\title{
Secondary Forest Succession in the Mata Atlantica, Brazil: Floristic and Phytosociological Trends
}

\author{
Alexandre Siminski, ${ }^{1}$ Alfredo Celso Fantini, ${ }^{1}$ Raymond Paul Guries, ${ }^{2}$ \\ Ademir Roberto Ruschel, ${ }^{3}$ and Maurício Sedrez dos Reis ${ }^{1}$ \\ ${ }^{1}$ Núcleo de Pesquisas em Florestas Tropicais, Pós-Graduação em Recursos Genéticos Vegetais, Universidade Federal de Santa Catarina \\ (UFSC), Rodovia Admar Gonzaga 1346, Itacorubi, Florianópolis, SC 88034-900, Brazil \\ ${ }^{2}$ Department of Forest and Wildlife Ecology, University of Wisconsin-Madison, 1630 Linden Drive, Madison, WI 53706, USA \\ ${ }^{3}$ Embrapa-Cepatu, Belém, PA 66095-100, Brazil
}

Correspondence should be addressed to Alfredo Celso Fantini, afantini@cca.ufsc.br

Received 28 January 2011; Accepted 9 March 2011

Academic Editors: D. Loustau and A. D. Steinman

Copyright (C) 2011 Alexandre Siminski et al. This is an open access article distributed under the Creative Commons Attribution License, which permits unrestricted use, distribution, and reproduction in any medium, provided the original work is properly cited.

This study aimed at understanding the dynamics of ecological processes and the use of secondary forests in Santa Catarina state (Brazil). The data base for these studies was formed through forest inventories carried out in the three forest types of the state. The results of this study demonstrate that the patterns of diversity are very similar among the three forest types; however, the species compositions among the types are quite different. A total of 343 woody species belonging to 73 families were found in the $24,000 \mathrm{~m}^{2}$ sampling area, revealing the potential role of secondary forest in the conservation of biodiversity at the landscape scale. As expected, a small set of pioneer species dominates young secondary forests with shade-tolerant species becoming structurally important after 30 years. The patterns of forest structure and species diversity observed in study largely conform to the postagricultural secondary succession observed for many tropical forests.

\section{Introduction}

The continuing loss of primary tropical forests makes secondary forests increasingly important for maintaining biodiversity across large forested landscapes [1-4], while also providing for environmental services and sustainable economic development [5-7]. Today, less than 25\% of the original forest area in Santa Catarina state (Brazil) remains, mostly as small fragments of secondary forest in a mosaic intermixed with other land uses [8]. Most secondary forests in Santa Catarina and throughout much of the Mata Atlântica are privately held and result from fallow rotations in a dynamic agricultural system [9]. The recovery potential of such secondary forests is influenced by many interacting factors including the length and intensity of past land use, parcel size, soil conditions and landscape position, local climate regimes, proximity to forest seed sources, the presence or absence of seed banks, and dispersal agents, and interactions among regenerating species [10-16].
The important roles that secondary forests can play in conservation and economic development are poorly understood in Brazil. Even well-intentioned Brazilian laws governing forest protection and use can be counter-productive to conservation. Recent laws that define a "forest" in terms of arbitrary structural characteristics have led small farmers to shorten fallow periods so that secondary forest succession does not proceed to the point where it is recognized as "forest". This avoids federal/state restrictions on forest harvesting or land conversion [17] with the result that secondary forests which could contribute to biodiversity conservation while providing a range of nontimber forest products are now less likely to develop. The landscape increasingly is held only in the earliest stages of forest succession before being cropped again.

We focused on the successional dynamics of secondary forests in the youngest age classes that prevail across this landscape in order to suggest alternative definitions of "forest" that recognize their important values in conservation 
and economic development. In this paper we describe the regeneration patterns that occur in secondary forests of Santa Catarina on parcels having diverse cultivation histories and variable fallow periods. Anthropogenic disturbances such as forest clearing for agriculture are generally more intensive/severe than those arising from natural disturbances, but early stages of forest succession appear to be dominated in both instances by a small number of common woody species in well-defined stages typical of large gaps $[16,18,19]$. The specific objectives of this study were to examine the patterns of species diversity and abundance as well as the transitions during which woody species are added and removed during the early phases of secondary forest succession.

\section{Methods}

2.1. Study Areas. The study was carried out on 48 small farms in the counties of Anchieta, Garuva, Concórdia, Três Barras, Caçador and São Pedro de Alcântara, Santa Catarina, Brazil (Figure 1). These areas included three different vegetation formations common to the Mata Atlântica of Santa Catarina, seasonal deciduous forest, mixed ombrophylous forest and dense ombrophylous forest [20] recognized and protected by Brazilian Law no. 11.426/2006.

2.1.1. Seasonal Deciduous Forest (SDF). This forest type occurs in the Uruguay River basin at elevations ranging from 200 to 600 meters with an annual precipitation of $1.800 \mathrm{~mm} / \mathrm{yr}$ [21-24]. It is characterized by a closed canopy dominated by Lauraceae and emergent deciduous species, mostly Fabaceae and a subcanopy dominated by Sorocea bonplandii, Gynnanthes concolor and Trichilia species; epiphytes are poorly represented in comparison with the dense ombrophylous forest [21].

2.1.2. Mixed Ombrophylous Forest (MOF). This forest type is distributed across plateaus at approximately 500 meters elevation upwards on slopes rising to 1600 meters above sea-level with annual precipitation ranging from 1,600 to $2,100 \mathrm{~mm}$ [20]. This moist forest is characterized by a supercanopy dominated by Araucaria angustifolia, with subcanopy layers rich in species of Lauraceae, Myrtaceae, and Fabaceae $[25,26]$.

2.1.3. Dense Ombrophylous Forest (DOF). This forest was formerly common in coastal regions at elevations below 500 meters. The forest is characterized by hot temperatures, heavy rainfall (annual precipitation ranges from 1,500 to $2,000 \mathrm{~mm}$ ) and an evergreen canopy dominated by Lauraceae and Myrtaceae with an abundance of epiphytes and palms $[18,26]$.

2.2. Data Collection and Analysis. We inventoried a total of eighty $10 \mathrm{~m} \times 10 \mathrm{~m}$ plots in the dense ombrophylous forest (Garuva and São Pedro de Alcântara sites), and forty $20 \mathrm{~m} \times$ $10 \mathrm{~m}$ plots in each of the seasonal deciduous forest (Anchieta and Concórdia sites), and mixed ombrophylous forest (Três Barras and Caçador sites) types (a total of 160 plots covering $\left.24,000 \mathrm{~m}^{2}\right)$. Based on physiognomic features, together with land ownership information, we sited 160 plots distributed across four successional stages (after Klein [18]: shrubby stage (0-8 years), small tree stage ( $8-15$ years), Arboreal Stage (15-30 years) and advanced arboreal stage (30-60 years)). All woody plants taller than $1.5 \mathrm{~m}$ were identified and measured for $\mathrm{DBH}$ (diameter at breast height $=1.3 \mathrm{~m}$ ) and total height. Plant identifications were made with reference to the Angiosperm Phylogeny Group (APG) classification updated in APG II [27], and the expertise of Prof. Ademir Reis (Federal University of Santa Catarina and Curator of the Barbosa Rodriguez Herbarium) and Prof. Marcos Sobral (BHCB-Herbarium of the Botany Department at the Federal University of Minas Gerais).

Species richness (number of species), Shannon-Wiener's index of diversity $\left(H^{\prime}\right)$, evenness $(J)$, Simpson's diversity index $(D)$, "importance value" (IV) and absolute frequency were estimated using the FITOPAC 1 software package [28]. Rarefaction curves (Sobs) constructed using 100 randomized orders and the Chao1, Jackknife and Bootstrap estimators were derived using EstimateS 6.0 software [29]. Differences in values of stem density and basal area among stages were tested by one-way ANOVA with Tukey's multiple comparison tests using the STATISTICA 6.0 software package [30].

\section{Results}

A total of 13,548 woody plants were identified on the 160 plots, representing 343 species from 73 families (the appendix). The most common families represented (number of species in parentheses) were the Myrtaceae (38), Fabaceae (33), Asteraceae (27), Lauraceae (27), Rubiaceae (20) and Melastomataceae (17). Forty-four species were common to all three forest types, representing around 13\% of the species total, leading to a similarity estimate of about $10 \%$.

The dense ombrophylous forest (DOF) type contained $66 \%$ (230) of all the species recorded for all forest types. Our sampling design placed more but smaller plots in the DOF formation and this likely introduced a slight bias towards finding more species in this forest type. When the differences in plot number and area sampled are accounted for (DOF2 in Table 2), we estimate that the dense ombrophylous forest type would still include 59\% (204) of the species identified. The seasonal deciduous forest and mixed ombrophylous forest types were very similar in their estimated species richness but markedly less rich than the dense ombrophylous forest type.

The secondary succession process in all three forest types was characterized by an increase in (1) species richness, (2) evenness scores, and (3) Shannon-Wiener diversity index values. Only the Simpson's diversity index values decreased over time during the formation of taxonomically more diverse and structurally more complex communities (Table 2).

Despite the relatively high species richness that our sampling revealed in these secondary forests, the rarefaction curves (Sobs) and other estimators of richness (Chao1, Jack2 and Bootstrap; Figure 2) suggest that our sampling only found about three-fourths (75\% in SDF, $71 \%$ in MOF and 


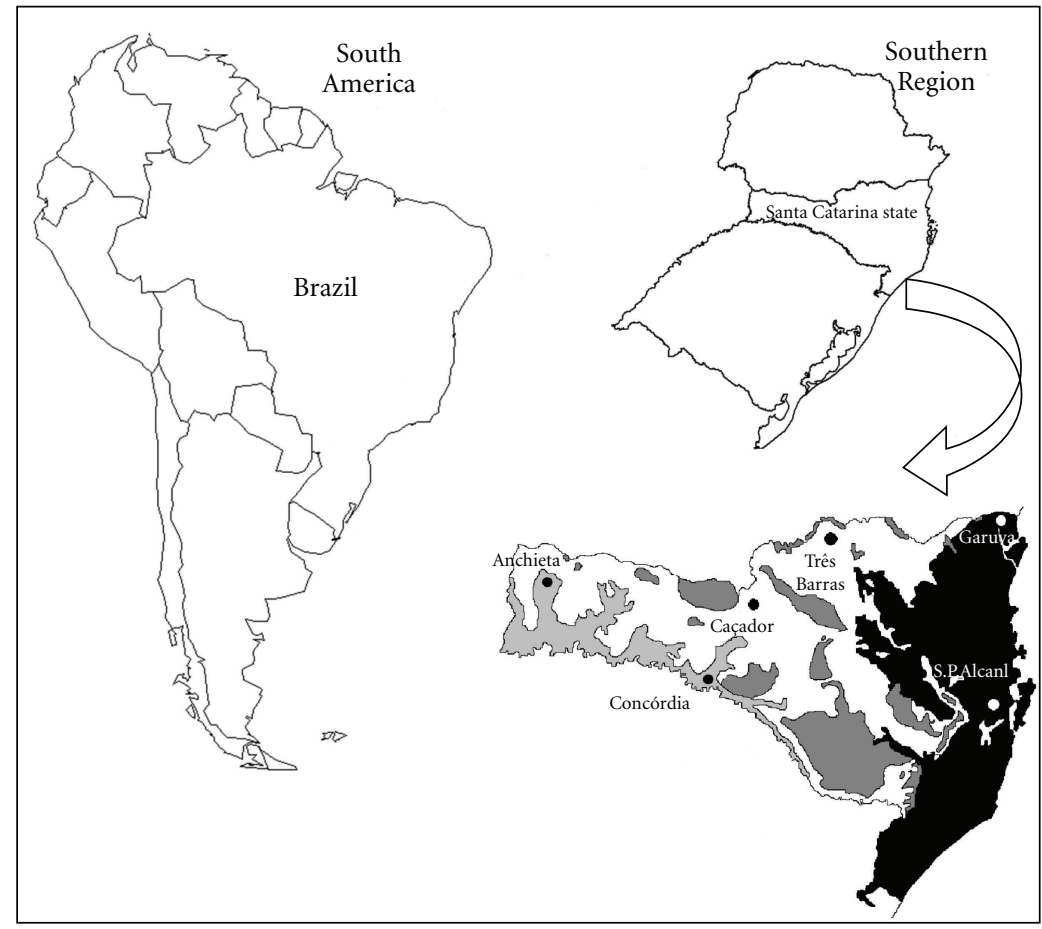

Dense Ombrophylous forest

$\square$ Campos de altitude

Mixed Ombrophylous forest

$\square$ Seasonal Deciduous forest

Figure 1: Santa Catarina phytogeographic map, adapted from Klein [25].

TABle 1: Family and species richness and the number of species common to three forest types in the Mata Atlântica of Santa Catarina, Brazil.

\begin{tabular}{lccccc}
\hline & SDF & MOF & DOF & All forests \\
\hline Families & 46 & 45 & 55 & 73 \\
Species & 135 & 135 & 230 & 343 \\
\hline \multirow{2}{*}{ Common species } & SDF/MOF & MOF/DOF & DOF/SDF & SDF/MOF/DOF \\
& 14 & 19 & 20 & 44 \\
\hline
\end{tabular}

$78 \%$ in DOF) of the maximum number of species expected to be present in these forest types.

Species composition for all three forest types changed with changes in the length of the preceding fallow period (Table 3). Although all three forests are marked by high species diversity, a few species dominate each stage of succession as demonstrated by the large Importance Values (Table 3), but this tendency decreases with increasing succession. As an example, the shrubby stage of all three forest types is dominated by Baccharis dracunculifolia and a few other species, while the small tree stage in the MOF and DOF types is dominated by Myrsine coriaceae and a few other species Nectandra lanceolata (SDF), Mimosa scabrela (MOF) and Miconia cinnamomifolia (DOF) dominated the Arboreal Stage and species such as Nectandra megapotamica (SDF), Ocotea puberula (MOF) and Hyeronima alchorneoides (DOF) dominated the advanced arboreal stage of each type.

During the early stages of secondary succession, species such as Trema micrantha (SDF) and Tibouchina trichona
(DOF) had low absolute frequencies but they had high densities and/or dominance when they occurred. In the advanced arboreal stages typical understory species such as Cupania vernalis, Allophylus edulis (SDF and MOF) and Euterpe edulis (DOF) all had high frequencies of occurrence. Most species with large Importance Values were common or abundant in only one or two successional stages but were quickly replaced by other species during succession. However, a few persisted, for example, Tibouchina pulchra was common in three successional stages in the DOF.

\section{Discussion}

The sampling method used identified those species most common to the early successional stages of secondary forest regeneration in three forest types of the Mata Atlântica. The high number of species (230) found in the dense ombrophylous forest was not unexpected, as this forest type represents some $82 \%$ of all arboreal species found in Santa Catarina [26]. 


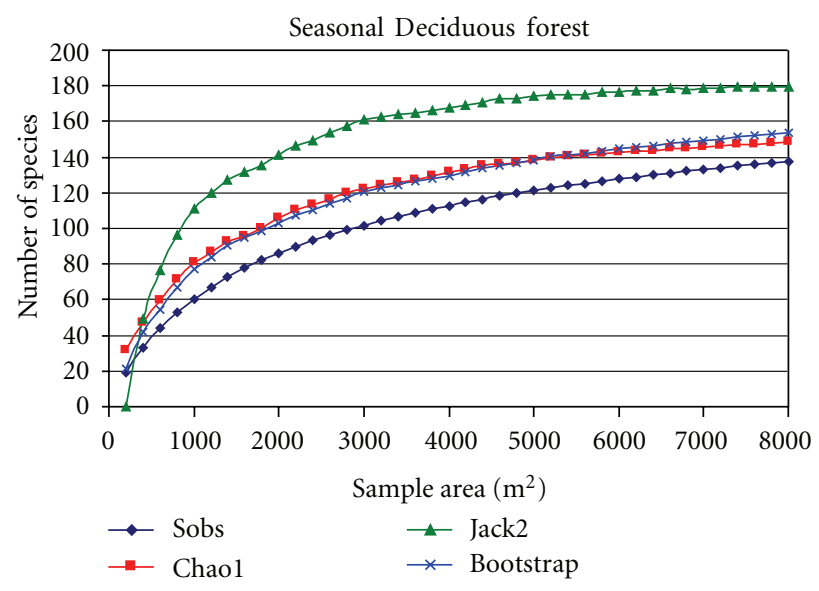

(a)

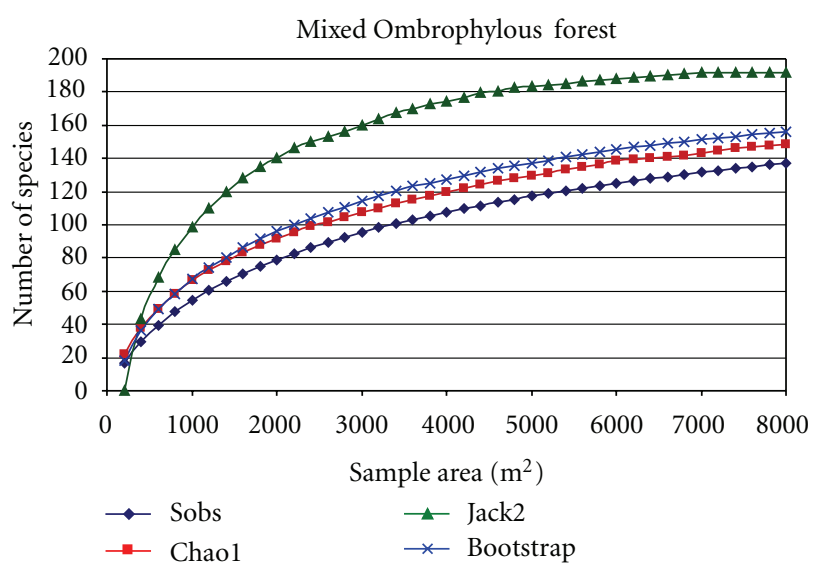

(b)

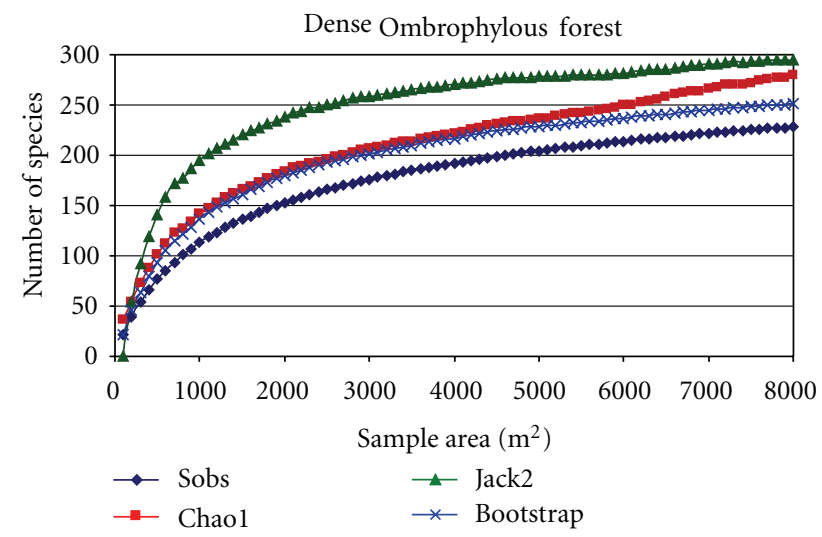

(c)

Figure 2: Sample-based rarefaction curves (Sobs) and species richness estimators (Chao1, Jack2 and Bootstrap) for three forest types in the Mata Atlântica.

Diversity and especially species richness increased over time since abandonment, similar to what has been found in other studies of swidden fields, abandoned plantations and pasture [13, 31-34].

The peak in the numbers of species found in Arboreal Stages of all forest types is consistent with the "intermediate disturbance hypothesis" [35], that during the early stages of secondary forest succession a few pioneer species dominate but are replaced over time by species better adapted to the increasingly competitive environments that develop. Species diversity is often greatest during mid-successional stages that contain both early and late successional species. In all three forest types studied here, the Arboreal Stage (15-30 years) had a richness that was greater than for any other stage in the SDF and MOF types and equal in richness to the advanced arboreal stage of the DOF type.

Species richness is only one component of diversity but because it gives the same weight to all species irrespective of their relative abundance it is strongly influenced by the number of rare species. Evenness, another component of diversity, is strongly influenced by the relative frequencies of dominant species $[36,37]$. We use different measures of diversity to emphasize different diversity patterns that reflect different ecological processes.

Our Shannon-Wiener diversity estimates were similar to, or slightly higher than, those of other studies of comparably aged Mata Atlântica forests. For example, Oliveira [34] and Torezan [38] estimated $H^{\prime}=2.51$ nats/ind in five-year-old early successional stands; Pessoa et al. [39] estimated $H^{\prime}=$ 3.66 nats/ind in a thirty-year-old pole stand; and Oliveira [34] estimated $H^{\prime}=3.33$ nats/ind in a twenty-five-yearold stand and $H^{\prime}=3.10$ nats/ind in a fifty-year-old area stand. All these studies were conducted on sites with land use histories characterized by shifting cultivation.

The Shannon index is a common diversity estimate reported in the literature ranging from 3.26 to 4.36 for the mature forests of these types [40-46]. The higher values reported here for all stages of our study in relation to values reported in the literature may be due to the sampling method and criteria used for plant inclusion. Also our use of more plots, especially noncontiguous plots, likely raised the levels of beta diversity.

Values of the Shannon index can be influenced by the number of species with intermediate values of relative 
TABLe 2: Phytosociological indices estimated for four successional stages in three Mata Atlântica forest formations; SDF: seasonal deciduous forest, MOF: mixed ombrophylous forest, DOF: dense ombrophylous forest; $H^{\prime}$ is Shannon-Wiener's index of diversity, $J^{\prime}$ is evenness, $D$ is Simpson's index.

\begin{tabular}{|c|c|c|c|c|c|c|c|c|}
\hline $\begin{array}{l}\text { Forest } \\
\text { Type }\end{array}$ & Successional stage & $\begin{array}{l}\text { Plots } \\
\text { (no.) }\end{array}$ & $\begin{array}{l}\text { Sampling } \\
\text { area }\left(\mathrm{m}^{2}\right)\end{array}$ & $\begin{array}{c}\text { Species } \\
\text { richness (no.) }\end{array}$ & $\begin{array}{c}\text { Family } \\
\text { richness (no.) }\end{array}$ & $\begin{array}{c}H^{\prime} \text { a } \\
\text { (nats/ind) }\end{array}$ & $J^{\prime}$ & $D$ \\
\hline \multirow{5}{*}{ SDF } & Shrubby (0-8 y) & 10 & 2000 & 45 & 24 & 2.08 & 0.400 & 0.335 \\
\hline & Small trees $(8-15 y)$ & 10 & 2000 & 72 & 32 & 3.25 & 0.760 & 0.076 \\
\hline & Arboreal (15-30y) & 10 & 2000 & 94 & 40 & 3.98 & 0.870 & 0.026 \\
\hline & $\begin{array}{l}\text { Advanced arboreal } \\
(30-60 y)\end{array}$ & 10 & 2000 & 84 & 33 & 3.70 & 0.840 & 0.036 \\
\hline & All stages & & 8000 & 135 & 46 & 4.00 & 0.817 & 0.034 \\
\hline \multirow{5}{*}{ MOF } & Shrubby (0-8 y) & 10 & 2000 & 46 & 19 & 2.74 & 0.719 & 0.122 \\
\hline & Small trees $(8-15 y)$ & 10 & 2000 & 51 & 22 & 3.16 & 0.797 & 0.069 \\
\hline & Arboreal (15-30y) & 10 & 2000 & 95 & 41 & 3.76 & 0.822 & 0.036 \\
\hline & $\begin{array}{l}\text { Advanced arboreal } \\
(30-60 y)\end{array}$ & 10 & 2000 & 81 & 34 & 3.57 & 0.809 & 0.052 \\
\hline & All stages & & 8000 & 135 & 45 & 3.92 & 0.799 & 0.035 \\
\hline \multirow{5}{*}{ DOF } & Shrubby $(0-8$ y) & 20 & 2000 & 80 & 30 & 3.09 & 0.705 & 0.073 \\
\hline & Small trees $(8-15 y)$ & 20 & 2000 & 116 & 43 & 3.35 & 0.700 & 0.058 \\
\hline & Arboreal (15-30 y) & 20 & 2000 & 163 & 51 & 4.24 & 0.829 & 0.025 \\
\hline & $\begin{array}{l}\text { Advanced arboreal } \\
(30-60 y)\end{array}$ & 20 & 2000 & 161 & 48 & 4.42 & 0.867 & 0.019 \\
\hline & All stages & & 8000 & 230 & 55 & 4.28 & 0.787 & 0.031 \\
\hline \multirow{5}{*}{ DOF2 } & Shrubby (0-8 y) & 10 & 1000 & 75 & 29 & 3.03 & 0.701 & 0.081 \\
\hline & Small trees $(8-15 y)$ & 10 & 1000 & 92 & 35 & 3.32 & 0.755 & 0.076 \\
\hline & Arboreal (15-30y) & 10 & 1000 & 132 & 46 & 4.16 & 0.852 & 0.026 \\
\hline & $\begin{array}{l}\text { Advanced arboreal } \\
(30-60 y)\end{array}$ & 10 & 1000 & 134 & 43 & 4.42 & 0.902 & 0.016 \\
\hline & All stages & & 4000 & 204 & 53 & 4.22 & 0.794 & 0.031 \\
\hline
\end{tabular}

${ }^{a}$ Estimated using the natural logarithm (base $e$ ).

abundance [47], and may introduce some variation in the estimates of species richness of communities [48]. As the value of Simpson's $D$ has an inverse relationship with the indices of Shannon and evenness [49], the value of $D$ decreases along successional stages.

The floristic composition of secondary forest formations described in this study was very similar with respect to genera reported for other studies in the Mata Atlântica of southeastern Brazil (Table 4).

\section{Conclusion}

The floristic diversity observed for the forests we surveyed largely agree with patterns of post-agricultural secondary forest succession observed for many other neotropical forests. The chronosequence covers a relatively large range of successional ages (0-60 years) that results in large changes in species diversity and composition despite a limited geographical sampling. This result suggests that the patchy mosaic of secondary forests in Santa Catarina has a high potential for biodiversity conservation. Whether it can also provide for economic development in the way of nontimber forest products would depend upon the life histories of individual species and any rules instituted to guide their sustainable management.

The results of this study demonstrate that the patterns of secondary succession appear very similar among the three forest types with respect to changes in species richness and other measures. However, the species composition among the types, while quite similar in the earliest shrubby stage, diverges during succession, with the largest differences noted between the DOF and the other two types. As expected, and despite high species richness, a small set of "pioneer species" dominates the shrubby and small tree stages until about age 15. After that time, more shade-tolerant species increase during the Arboreal and advanced arboreal stages of forest succession, but only a small number become structurally important after $25-30$ years. 
TABLE 3: Woody species most characteristic of four secondary forest successional stages in the Mata Atlântica of Santa Catarina.

\begin{tabular}{|c|c|c|c|c|}
\hline Successional stage & $\begin{array}{c}\text { Dominan } \\
\text { species }^{\mathrm{a}}\end{array}$ & Five most important species & IV value ${ }^{b}$ & $\begin{array}{c}\text { Successional } \\
\text { position }^{c}\end{array}$ \\
\hline \multicolumn{5}{|c|}{ Seasonal deciduous forest (SDF) } \\
\hline Shrubby & 6 & $\begin{array}{l}\text { Baccharis calvensces, B. dracunculifolia, B. elaeagnoides, Anonna } \\
\text { silvatica, Schinus terebinthifolius. }\end{array}$ & 137 & $\mathrm{E}$ \\
\hline Small trees & 9 & $\begin{array}{l}\text { Baccharis dracunculifolia, Dalbergia frutescens, Schinus } \\
\text { terebinthifolius, Solanum mauritianum, Trema micrantha }\end{array}$ & 108 & $\mathrm{E}$ \\
\hline Arboreal & 16 & $\begin{array}{l}\text { Alchornea triplinervia, Aloysia virgata, Apuleia leiocarpa, } \\
\text { Dahlstedtia pinnata, Nectandra lanceolata }\end{array}$ & 64 & M \\
\hline Advanced arboreal & 12 & $\begin{array}{l}\text { Alchornea triplinervia, Cupania vernalis, Nectandra lanceolata, } \\
\text { N. megapotamica, Parapiptadenia rigida. }\end{array}$ & 100 & M-L \\
\hline \multicolumn{5}{|c|}{ Mixed ombrophylous forest (MOF) } \\
\hline Shrubby & 6 & $\begin{array}{l}\text { Baccharis dracunculifolia, B. semiserrata, Eupatorium } \\
\text { vauthierianum, Piptocarpha angustifolia, Solanum mauritianum. }\end{array}$ & 137 & $\mathrm{E}$ \\
\hline Small trees & 9 & $\begin{array}{l}\text { Baccharis dracunculifolia, Matayba elaeagnoides, Mimosa } \\
\text { scabrella, Myrsine coriaceae, Sapium glandulatum. }\end{array}$ & 100 & $\mathrm{E}$ \\
\hline Arboreal & 11 & $\begin{array}{l}\text { Clethra scabra, Matayba elaeagnoides, Mimosa scabrella, Ocotea } \\
\text { puberula, O. pulchella. }\end{array}$ & 97 & M \\
\hline Advanced arboreal & 11 & $\begin{array}{l}\text { Clethra scabra, Cupania vernalis, Matayba elaeagnoides, Ocotea } \\
\text { porosa, O. puberula. }\end{array}$ & 108 & $\mathrm{M}-\mathrm{L} / \mathrm{U}$ \\
\hline \multicolumn{5}{|c|}{ Dense ombrophylous forest (DOF) } \\
\hline Shrubby & 6 & $\begin{array}{l}\text { Baccharis dracunculifolia, Dodonaea viscosa, Leandra dasytricha, } \\
\text { Myrsine coriaceae, Tibouchina trichotona. }\end{array}$ & 115 & $\mathrm{E}$ \\
\hline Small trees & 8 & $\begin{array}{l}\text { Miconia cabucu, M. cinnamomifolia, M. rigidiuscula, Myrsine } \\
\text { coriaceae, Tibouchina pulchra. }\end{array}$ & 133 & E-M \\
\hline Arboreal & 17 & $\begin{array}{l}\text { Hyeronima alchorneoides, Miconia cabucu, M. cinnamomifolia, } \\
\text { Myrsine coriaceae, Tibouchina pulchra }\end{array}$ & 83 & M \\
\hline Advanced arboreal & 20 & $\begin{array}{l}\text { Euterpe edulis, Marlieria eugeniopsioides, Miconia } \\
\text { cinnamomifolia, Psychotria longipes, Tibouchina pulchra. }\end{array}$ & 44 & $\mathrm{M}-\mathrm{L} / \mathrm{U}$ \\
\hline
\end{tabular}

${ }^{\mathrm{a}}$ Dominant species are defined as those species whose summed importance values, when ranked from the highest to lowest, contained $50 \%$ of the total for a given stand, after Finegan [14].

${ }^{b}$ IV: Importance Value, here we include the proportion (from a base of 300) accounted for by the five most important species.

${ }^{\mathrm{c}}$ Successional position (E: early successional, M: mid successional, L: late successional, U: understory) of the five most important species.

TABLE 4: Plant genera with a high frequency of occurrence in secondary forests of the Mata Atlântica in Southern and Southeastern Brazil.

\begin{tabular}{lll}
\hline Forest Formation & Frequent botanic genus & Authors \\
\hline $\begin{array}{l}\text { Seasonal deciduous } \\
\text { forest }\end{array}$ & $\begin{array}{l}\text { Baccharis, Alchornea, Casearia, Inga, Solanum, Nectandra, Ocotea, } \\
\text { Ilex, Allophylus, Apuleia, Cedrela, Cupania, Lonchocarpus, Luehea, } \\
\text { Machaerium, Sorocea and Trema. }\end{array}$ & $\begin{array}{l}\text { Vaccaro and Longhi [50]; Rondon-Neto et al. } \\
\text { [51]; Andreis et al. [52]; Hack et al. [53]. }\end{array}$ \\
\hline $\begin{array}{l}\text { Mixed ombrophylous } \\
\text { forest }\end{array}$ & $\begin{array}{l}\text { Baccharis, Casearia, Chethra, Ilex, Solanum, Nectandra, Ocotea, } \\
\text { Myrsine, Matayba, Piptocarpha, Sapium, Vernonia, Allophylus, }\end{array}$ & $\begin{array}{l}\text { Rondon-Neto et al. [54]; Pezzatto et al. [55]; } \\
\text { Zanthoxylum and Capsicodendron. }\end{array}$ \\
\hline \multirow{2}{*}{$\begin{array}{l}\text { Dense ombrophylous et al. [56]; Ramos and Boldo [57]. } \\
\text { forest }\end{array}$} & $\begin{array}{l}\text { Baccharis, Tibouchina, Myrsine, Cecropia, Alchornea, Solanum, } \\
\text { Miconia, Nectandra, Ocotea, Jacaranda, Chethra, Ilex, Cedrela, } \\
\text { Cupania, Psychotria, Euterpe, Guarea and Matayba. }\end{array}$ & $\begin{array}{l}\text { Klein [18]; Tabarelli and Mantovani [33]; } \\
\text { Mantovani et al. [59]; Schorn and Galvão [60]; } \\
\text { Liebsh et al. [61]. }\end{array}$ \\
\hline
\end{tabular}

A chronosequence approach only allows us to infer successional changes because we do not analyze the underlying processes mediating these changes on our sites (growth, mortality, and recruitment). Thus, we suggest that long-term permanent plots be established in this region, to improve our understanding of secondary forest dynamics, while also creating a framework for future comparative studies of the role of ecological processes and mechanisms in different successional stages.

\section{Appendix}

See Table 5 . 
TABLE 5: List of families, species and number of individuals in four successional stages (SR: shrubby, ST: small trees, AR: arboreal and AA: advanced arboreal) in the Mata Atlântica forest formations, Brazil; SDF: seasonal deciduous forest, MOF: mixed ombrophylous forest, DOF: dense ombrophylous forest.

\begin{tabular}{|c|c|c|c|c|c|c|c|c|c|c|c|c|}
\hline \multirow{2}{*}{ Botanic family/species } & \multicolumn{4}{|c|}{ SDF } & \multicolumn{4}{|c|}{ MOF } & \multicolumn{4}{|c|}{ DOF } \\
\hline & SR & ST & $\mathrm{AR}$ & $\mathrm{AA}$ & SR & ST & $\mathrm{AR}$ & $\mathrm{AA}$ & SR & ST & $\mathrm{AR}$ & $\mathrm{AA}$ \\
\hline \multicolumn{13}{|l|}{ Adoxaceae (Caprifoliaceae) } \\
\hline Sambucus australis Cham. \& Schltdl. & & & 1 & & & & & & & & & \\
\hline \multicolumn{13}{|l|}{ Anacardiaceae } \\
\hline Lithraea brasiliensis March. & & & & & 1 & 1 & 38 & & & & & \\
\hline Schinus molle L. & & & & & 3 & 5 & 3 & & & & & \\
\hline Schinus terebinthifolius Raddi & 21 & 51 & 8 & & 22 & 40 & 41 & 1 & & & 1 & \\
\hline Tapirira guianensis Aubl. & & & & & & & & & & 4 & & \\
\hline \multicolumn{13}{|l|}{ Annonaceae } \\
\hline Annona glabra L. & & & & & & & & & & & 3 & 4 \\
\hline Duguetia lanceolata A.St.-Hil. & & & & & & & & & 1 & & 2 & 5 \\
\hline Guatteria australis A.St.-Hil. & & & & & & & & & 1 & 1 & 1 & 3 \\
\hline Annona rugulosa Schl. & & & 6 & 3 & 3 & 11 & 21 & 10 & & & & \\
\hline Annona sericea R.E.Fries & & & 1 & & & & & & & 6 & 9 & 20 \\
\hline Annona sylvatica A.St.-Hil. & 23 & 2 & 9 & 4 & & & & & & 7 & 9 & 6 \\
\hline Annona sp. & & & & & & & & & & & 1 & 2 \\
\hline Xylopia brasiliensis Spreng. & & & & & & & & & & & 34 & 27 \\
\hline \multicolumn{13}{|l|}{ Apocynaceae } \\
\hline Aspidosperma parvifolium A.DC. & 5 & 2 & 4 & 1 & & & & & & & 2 & 6 \\
\hline Rauvolfia sellowii Müll.Arg. & & 1 & 3 & 7 & & & & & & & & \\
\hline Tabernaemontana catharinensis A. DC. & & & & & & & & & & 2 & 3 & 5 \\
\hline \multicolumn{13}{|l|}{ Aquifoliaceae } \\
\hline Ilex brevicuspis Reissek & & & & & & & 1 & 1 & & & 1 & 4 \\
\hline Ilex dumosa Reissek & & & & & & & & & & & 6 & 1 \\
\hline Ilex microdonta Reissek & & & & & & & 2 & & & & 3 & 1 \\
\hline Ilex paraguariensis A.St.-Hil. & 2 & 2 & 43 & & & 5 & 73 & 27 & & & & \\
\hline Ilex theezans Mart. ex Reissek & & & & & & 2 & 7 & 1 & 1 & & 9 & 7 \\
\hline \multicolumn{13}{|l|}{ Araliaceae } \\
\hline $\begin{array}{l}\text { Schefflera angustissima (Marchal) } \\
\text { Frodin }\end{array}$ & & & & 1 & & & & 1 & & 1 & 14 & 3 \\
\hline $\begin{array}{l}\text { Schefflera morototoni (Aubl.) Maguire, } \\
\text { Stey. \& Frod }\end{array}$ & & & & & 3 & 5 & 3 & & & & & \\
\hline \multicolumn{13}{|l|}{ Araucariaceae } \\
\hline Araucaria angustifolia (Bertol.) Kuntze & & & & & & 2 & 2 & 3 & & & & \\
\hline \multicolumn{13}{|l|}{ Arecaceae } \\
\hline Bactris setosa Mart. & & & & & & & & & & 5 & 28 & 19 \\
\hline Euterpe edulis Mart. & & & & & & & & & & & 18 & 131 \\
\hline Geonoma gamiova Barb. & & & & & & & & & & 1 & 6 & 41 \\
\hline Geonoma schottiana Mart. & & & & & & & & & & 2 & 2 & 2 \\
\hline $\begin{array}{l}\text { Syagrus romanzoffiana (Cham.) } \\
\text { Glassman }\end{array}$ & & & & & & 2 & & & & & & \\
\hline \multicolumn{13}{|l|}{ Asteraceae } \\
\hline Asteracea sp1 & & & & & & & & & 1 & & & \\
\hline Asteracea sp2 & & 1 & & & & & & & & & & \\
\hline Baccharis calvensces A.P. Candole & 16 & 10 & & & 14 & 2 & 1 & & 79 & 23 & 2 & \\
\hline Baccharis dentata (Vell.) G.M.Barroso & & & & & 1 & & & & & & & \\
\hline
\end{tabular}


TABle 5: Continued.

\begin{tabular}{|c|c|c|c|c|c|c|c|c|c|c|c|c|}
\hline \multirow{2}{*}{ Botanic family/species } & \multicolumn{4}{|c|}{ SDF } & \multicolumn{4}{|c|}{ MOF } & \multicolumn{4}{|c|}{ DOF } \\
\hline & SR & ST & $\mathrm{AR}$ & $\mathrm{AA}$ & SR & ST & $\mathrm{AR}$ & $\mathrm{AA}$ & SR & ST & $\mathrm{AR}$ & $\mathrm{AA}$ \\
\hline Baccharis dracunculifolia DC. & 307 & 72 & 1 & & 203 & 114 & 11 & & 81 & 22 & 1 & \\
\hline Baccharis elaeagnoides Steud. & 14 & 11 & & & 23 & 2 & & & 81 & 24 & & \\
\hline Baccharis erioclada DC. & & & & & 31 & & & & & & & \\
\hline Baccharis semiserrata DC. & 3 & & & & 8 & & & & & & & 5 \\
\hline Baccharis sp1 & 16 & 10 & & & & 1 & & & & & & \\
\hline Baccharis sp2 & & & & & & 1 & & & 81 & 32 & & \\
\hline Baccharis sp3 & & & & & 11 & & & & & & & \\
\hline Baccharis trimera (Less.) DC. & & & & & 4 & & & & & & & \\
\hline Baccharis uncinella DC. & & & & & 5 & & & & & & & \\
\hline Eupatorium serratum Spreng. & & & & & 3 & & & & & 9 & & \\
\hline Eupatorium $\mathrm{sp} 2$ & & & & & 32 & 7 & 2 & & & & & \\
\hline Eupatorium sp3 & & & & & & & & & 5 & & & \\
\hline Eupatorium sp1 & 2 & 1 & & & & & & & & & & \\
\hline Eupatorium vauthierianum DC. & & & & & 132 & 51 & & 13 & & & & \\
\hline Gochnatia polymorpha (Less.) Cabrera. & & & & & & 2 & & & & & & \\
\hline $\begin{array}{l}\text { Piptocarpha angustifolia Dusén ex } \\
\text { Malme }\end{array}$ & & & & 1 & 44 & 1 & 5 & & 11 & 20 & 13 & \\
\hline Piptocarpha tomentosa Baker. & & 1 & & & & & 6 & 1 & & 2 & 1 & \\
\hline Vernonia discolor (Spreng.) Less. & & & & 6 & 1 & 5 & 25 & 2 & 5 & 46 & 37 & 3 \\
\hline Vernonia petiolaris DC. & & & & & 27 & & & & & & & \\
\hline Vernonia puberula Less. & & & & & & & & & 13 & & & 1 \\
\hline Vernonia sp1 & & & & & 14 & 2 & 2 & & & & & \\
\hline Vernonia sp2 & & & & & & 7 & & & & & & \\
\hline Vernonia sp3 & & & & & & & & & & & 1 & \\
\hline \multicolumn{13}{|l|}{ Bignoneaceae } \\
\hline Jacaranda micrantha Cham. & & & 1 & 5 & 2 & 6 & 4 & 2 & 21 & 53 & 47 & 3 \\
\hline Jacaranda puberula Cham. & & & & & 1 & 1 & 3 & 1 & 3 & 37 & 12 & \\
\hline Tabebuia sp. & & & & & & & & & & & 6 & 2 \\
\hline Tabebuia umbellata (Sond.) Sandw. & 1 & 4 & 8 & 2 & & & & & & 3 & & \\
\hline \multicolumn{13}{|l|}{ Boraginaceae } \\
\hline Cordia ecalyculata Vell & 1 & 13 & 9 & 12 & & & & & & & & \\
\hline $\begin{array}{l}\text { Cordia americana (L.) Gottschling \& } \\
\text { J.S. Mill. }\end{array}$ & 3 & 2 & 3 & 5 & & & & & & & & \\
\hline \multicolumn{13}{|l|}{ Burseraceae } \\
\hline Protium kleinii Cuatrec. & & & & & & & & & & & 3 & 1 \\
\hline \multicolumn{13}{|l|}{ Cannabaceae (Ulmaceae) } \\
\hline Celtis triflora (Klotszch.)Miq. & & & & & & 4 & & & & & & \\
\hline Trema micrantha (L.) Blume & 8 & 186 & 4 & 1 & & 1 & & & 7 & 7 & 1 & \\
\hline \multicolumn{13}{|l|}{ Canellaceae } \\
\hline $\begin{array}{l}\text { Capsicodendron dinisii (Schwacke) } \\
\text { Occhioni }\end{array}$ & & & & & & 2 & 32 & 31 & & & & \\
\hline \multicolumn{13}{|l|}{ Cardiopteridaceae (Icacinaceae) } \\
\hline $\begin{array}{l}\text { Citronella paniculata (Mart.) } \\
\text { R.A.Howard }\end{array}$ & & & 2 & 14 & & & & & & & & \\
\hline
\end{tabular}


Table 5: Continued.

\begin{tabular}{|c|c|c|c|c|c|c|c|c|c|c|c|c|}
\hline \multirow{2}{*}{ Botanic family/species } & \multicolumn{4}{|c|}{ SDF } & \multicolumn{4}{|c|}{ MOF } & \multicolumn{4}{|c|}{ DOF } \\
\hline & SR & ST & $\mathrm{AR}$ & $\mathrm{AA}$ & SR & ST & $\mathrm{AR}$ & $\mathrm{AA}$ & SR & ST & $\mathrm{AR}$ & $\mathrm{AA}$ \\
\hline \multicolumn{13}{|l|}{ Caricaceae } \\
\hline Carica quercifolia (A.St.-Hil.) Hieron & & 2 & & & & & & & & & & \\
\hline \multicolumn{13}{|l|}{ Celastraceae } \\
\hline Maytenus muelleri Scheacke & & & 3 & 1 & & & 4 & 1 & & & & \\
\hline Maytenus robusta Reiss. & & & & & & & & & 1 & & 2 & 2 \\
\hline \multicolumn{13}{|l|}{ Chloranthaceae } \\
\hline Hedyosmum brasiliensis Mart. & & & & & & & & & 1 & 9 & 17 & \\
\hline \multicolumn{13}{|l|}{ Chrysobalanaceae } \\
\hline Hirtella hebeclada Moric. ex DC. & & & & & & & & & 1 & 2 & 3 & 8 \\
\hline \multicolumn{13}{|l|}{ Clethraceae } \\
\hline Clethra scabra Pers. & & 1 & 2 & & 14 & 24 & 29 & 22 & 2 & 10 & 60 & 7 \\
\hline \multicolumn{13}{|l|}{ Clusiaceae } \\
\hline Calophyllum brasiliense Cambess. & & & & & & & & & & & 4 & 1 \\
\hline Clusia parviflora (Saldanha) Enfler & & & & & & & & & & 9 & 24 & 7 \\
\hline $\begin{array}{l}\text { Garcinia gardneriana (Planch. \& } \\
\text { Triana) Zappi }\end{array}$ & & & & & & & & & 1 & & 5 & 8 \\
\hline \multicolumn{13}{|l|}{ Combretaceae } \\
\hline Terminalia cf. australis Cambess. & & & & & & & & & & 2 & 1 & \\
\hline
\end{tabular}

\section{Cunoniaceae}

Lamanonia speciosa (Camb.) L.B.

Smith.

$\begin{array}{llll}3 & 3 & 5 & 7\end{array}$

Lamanonia ternata Vell.

Weinmania humilis Engl.

Weinmannia paulliniifolia Pohl ex Ser.

Cyatheaceae

Alsophila sp.

Cyathea schanschin Mart.

Cyathea vestita Mart.

Dicksoniaceae

Dicksonia sellowiana Hook

Ebenaceae

Diospyros inconstans Jacq.

Elaeocarpaceae

Sloanea guianensis (Aubl.) Benth.

Erythroxylaceae

Erythroxyllum deciduum A.St.-Hil.

Erythroxylum cf. cuneifolium (Mart.)

O.E. Schulz

Erythroxylum myrsinites Mart.

$4 \quad 2$

\section{Euphorbiaceae}

Alchornea iricurana Casar.

Alchornea sidifolia Müll.Arg.

Alchornea triplinervia (Spreng.) Müll.

Arg.

1

1

Gymnanthes concolor Spreng.

$\begin{array}{llll}3 & & 2 & 2 \\ 1 & 5 & 23 & 28\end{array}$

$1 \quad 20 \quad 24$

$3 \quad 2$ 
Table 5: Continued.

\begin{tabular}{|c|c|c|c|c|c|c|c|c|c|c|c|c|}
\hline \multirow{2}{*}{ Botanic family/species } & \multicolumn{4}{|c|}{ SDF } & \multicolumn{4}{|c|}{ MOF } & \multicolumn{4}{|c|}{ DOF } \\
\hline & SR & ST & $\mathrm{AR}$ & $\mathrm{AA}$ & SR & ST & AR & $\mathrm{AA}$ & SR & ST & $\mathrm{AR}$ & $\mathrm{AA}$ \\
\hline Manihot grahami Hook. & 6 & 11 & 4 & 1 & & 10 & 2 & 1 & & & 5 & \\
\hline Pausandra morisiana (Casar.) Radlk. & & & & & & & & & & & 3 & 3 \\
\hline Pera glabrata (Schott) Baill & & & & & & & & & 1 & 4 & 53 & 25 \\
\hline Ricinus communis L. (exótica) & 2 & & & & & & & & & & & \\
\hline Sapium glandulosum (L.) Morong & 1 & 1 & 1 & & 6 & 20 & 18 & 11 & 1 & 7 & 1 & 1 \\
\hline $\begin{array}{l}\text { Sebastiania commersoniana (Baill.) } \\
\text { L.B. Smith \& R.J.Downs }\end{array}$ & 1 & 3 & 4 & 3 & & & & & & & & \\
\hline $\begin{array}{l}\text { Tetrorchidium rubrivenium Poepp. \& } \\
\text { Endl. }\end{array}$ & & & & & & & & & 120 & 21 & 27 & \\
\hline \multicolumn{13}{|l|}{ Phylanthaceae } \\
\hline Hyeronima alchorneoides Fr. Allem. & & & & & & & & & 32 & 58 & 78 & 39 \\
\hline Richeria australis Mull.Arg. & & & & & & & & & & & 1 & \\
\hline \multicolumn{13}{|l|}{ Fabaceae } \\
\hline $\begin{array}{l}\text { Abarema langsdorfii (Benth.) Barneby } \\
\text { \& J.W. Grimes }\end{array}$ & & & & & & & & & & 2 & 5 & \\
\hline Albizia polycephala (Benth.) Killip & & 5 & & & & & & & & & & \\
\hline Andira anthelmintica Benth. & & & 1 & & & & & & & 5 & & 1 \\
\hline Andira fraxinifolia Benth. & & & & & & & & & & & 1 & \\
\hline Apuleia leiocarpa (Vog.) Macbr. & 2 & & 25 & 11 & & & & & & & & \\
\hline Bauhinia forficata Link. & 2 & 11 & 5 & 19 & & & & & & & & \\
\hline Calliandra selloi (Spreng.) Macbr. & & 3 & 6 & 1 & & & & & & & & \\
\hline Copaifera trapezifolia Hayne & & & & & & & & & & & & 3 \\
\hline Dahlstedtia pinnata (Benth.) Malme & & 7 & 36 & & & & & & & & & \\
\hline Dalbergia frutescens Britton & 17 & 73 & 19 & 36 & & & & & & & & 2 \\
\hline $\begin{array}{l}\text { Enterolobium contortisiliquum (Vell.) } \\
\text { Morong. }\end{array}$ & & & & 1 & & & & & & & & \\
\hline Holocalyx balansae Micheli & & & & 2 & & & & & & & & \\
\hline Inga marginata Willd. & & 26 & 20 & 7 & & & & & 7 & 3 & 6 & 2 \\
\hline Inga sessilis (Vell.) Mart. & & & & & & & & & 4 & 2 & 2 & 1 \\
\hline Inga vera Willd. & & 11 & 7 & & & & & & & & & 1 \\
\hline Inga virescens Benth. & & & & & 1 & 1 & 3 & 18 & & & 1 & \\
\hline $\begin{array}{l}\text { Lonchocarpus campestris Mart. ex } \\
\text { Benth }\end{array}$ & 5 & 4 & 39 & 10 & & 29 & 17 & & 11 & 12 & & \\
\hline $\begin{array}{l}\text { Lonchocarpus guilleminianus (Tul.) } \\
\text { Malme }\end{array}$ & & 3 & 12 & 7 & & & 10 & 12 & 1 & & & \\
\hline Lonchocarpus sp. & & & 9 & 1 & & & & & & & 1 & \\
\hline Machaerium hirtum (Vell.) Stell Feld & & & & & & & & & & & & 2 \\
\hline Machaerium paraguariensis Hassler & 6 & 22 & 2 & & 1 & 2 & & 2 & 1 & & & \\
\hline Machaerium sp.1 & & 49 & & & & & & & & & & \\
\hline Machaerium stipitatum (DC.) Vog. & & 34 & 3 & 3 & & 24 & 13 & 1 & 3 & & 2 & 2 \\
\hline Mimosa bimucronata (DC.) O. Ktze. & & & & & & & & & 6 & 16 & 2 & 3 \\
\hline Mimosa scabrella Benth & & & & & 8 & 13 & 75 & 2 & & & & \\
\hline Myrocarpus frondosus Allem. & & 2 & & 3 & & & & & & & & \\
\hline Ormosia arborea (Vell.) Harms. & & & & & & & & & 1 & 1 & & \\
\hline Papilionaceae sp. & & & & & & & & & & & & 1 \\
\hline
\end{tabular}


TABle 5: Continued.

\begin{tabular}{|c|c|c|c|c|c|c|c|c|c|c|c|c|}
\hline \multirow{2}{*}{ Botanic family/species } & \multicolumn{4}{|c|}{ SDF } & \multicolumn{4}{|c|}{ MOF } & \multicolumn{4}{|c|}{ DOF } \\
\hline & SR & ST & $\mathrm{AR}$ & AA & SR & ST & $\mathrm{AR}$ & $\mathrm{AA}$ & SR & ST & $\mathrm{AR}$ & AA \\
\hline Parapiptadenia rigida (Benth.) Brenan & 5 & 11 & 15 & 37 & & & & & & & & \\
\hline Peltophorum dubium (Spreng.) Taub. & & & 1 & & & & & & & & & \\
\hline $\begin{array}{l}\text { Piptadenia gonoacantha (Mart.) } \\
\text { Macbr. }\end{array}$ & & & & & & & & & 6 & & & 3 \\
\hline Schizolobium parahyba (Vell.) Blake & & & & & & & 3 & & & & & \\
\hline Zollernia ilicifolia Vog. & & & & & & & & & 1 & & 9 & 4 \\
\hline \multicolumn{13}{|l|}{ Lamiaceae } \\
\hline Aegiphila sellowiana Cham. & & & & & & & 5 & 2 & & & 3 & 5 \\
\hline $\begin{array}{l}\text { Vitex megapotamica (Spreng.) } \\
\text { Moldenke }\end{array}$ & & & 2 & 3 & & & & & & & & 1 \\
\hline \multicolumn{13}{|l|}{ Lauraceae } \\
\hline $\begin{array}{l}\text { Cinnamomum amoenum (Nees) } \\
\text { Kosterm. }\end{array}$ & & & & & & & 1 & 1 & & & & \\
\hline Cryptocarya aschersoniana $\mathrm{Mez}$ & & & & & & & & 3 & & & & \\
\hline $\begin{array}{l}\text { Cryptocarya cf. moschata Nees et Mart. } \\
\text { ex Nees }\end{array}$ & & & & & & & & & & & & 10 \\
\hline $\begin{array}{l}\text { Endlicheria paniculata (Spreng.) } \\
\text { Macbride }\end{array}$ & & & 3 & 3 & & & & & & & & 8 \\
\hline Lauraceae sp1 & & 1 & 1 & & & & & & & & & \\
\hline Lauraceae sp2 & & & & & & & & & & & & 1 \\
\hline $\begin{array}{l}\text { Nectandra lanceolata Nees et Mart. ex } \\
\text { Nees }\end{array}$ & 2 & 28 & 67 & 73 & & 1 & 6 & 39 & & 6 & 12 & 11 \\
\hline Nectandra leucothyrsus Meissn. & & & & & & & & & & 1 & 2 & 3 \\
\hline Nectandra megapotamica Mez. & & 14 & 18 & 90 & & 6 & & 4 & 1 & 11 & 2 & 20 \\
\hline Nectandra membranacea (Sw.) Griseb. & & & & & & & & & & & 6 & 10 \\
\hline Nectandra oppositifolia Nees & & & & & & & & & 1 & 2 & 10 & 13 \\
\hline Nectandra rigida (Kunth) Nees & & & 1 & & & & & & & & & 2 \\
\hline Nectandra sp1 & & & & & & & & & & & & 1 \\
\hline Nectandra sp2 & & 1 & & & & & & & & & & \\
\hline Nectandra sp3 & & & & & & & & 6 & & & & \\
\hline Ocotea catharinensis $\mathrm{Mez}$ & & & & & & & & & & & & 1 \\
\hline Ocotea diospyrifolia (Meisn.) Mez & 1 & 4 & 13 & 29 & & & 2 & 2 & & & & 1 \\
\hline Ocotea odorifera (Vell.) Rohwer & & & 5 & 5 & & & & & & & & \\
\hline $\begin{array}{l}\text { Ocotea porosa (Nees \& C. Mart.) } \\
\text { Barroso }\end{array}$ & & & & & & 14 & 1 & 19 & & & & \\
\hline Ocotea puberula (Rich.) Nees & & 2 & 4 & & 7 & 16 & 53 & 38 & & 1 & & 2 \\
\hline Ocotea pulchella Mart. & & & 2 & 10 & 1 & 7 & 33 & 18 & & & 10 & \\
\hline Ocotea sp1 & & & & & & & & & & & & 3 \\
\hline Ocotea sp2 & & & & & & & 3 & & & & & \\
\hline Ocotea teleiandra (Meissn.) Mez & & & & & & & & & & & & 1 \\
\hline Persea americana Mill. & & & & 4 & & & & & & & & \\
\hline Persea major (Nees) L. E. Kopp & & & & & & & & 1 & & & & \\
\hline Persea sp. & & & & 2 & & & 1 & & & & & \\
\hline \multicolumn{13}{|l|}{ Loganiaceae } \\
\hline Strychnos brasiliensis (Spreng.) Mart. & & & & & & & 1 & 3 & & & 6 & 5 \\
\hline
\end{tabular}


Table 5: Continued.

\begin{tabular}{|c|c|c|c|c|c|c|c|c|c|c|c|c|}
\hline \multirow{2}{*}{ Botanic family/species } & \multicolumn{4}{|c|}{ SDF } & \multicolumn{4}{|c|}{ MOF } & \multicolumn{4}{|c|}{ DOF } \\
\hline & SR & ST & $\mathrm{AR}$ & AA & SR & ST & $\mathrm{AR}$ & AA & SR & ST & $\mathrm{AR}$ & $\mathrm{AA}$ \\
\hline \multicolumn{13}{|l|}{ Lythraceae } \\
\hline Lafoensia pacari A.St.-Hil. & & & & & & & 1 & & & & & \\
\hline \multicolumn{13}{|l|}{ Magnoliaceae } \\
\hline Magnolia ovata (A.St.-Hil.) Spreng. & & & & & & & & & & 2 & & 1 \\
\hline \multicolumn{13}{|l|}{ Malpighiaceae } \\
\hline Bunchosia maritima (Vell.) J. F. Macbr. & & & 2 & & & & & & & & & \\
\hline Byrsonima ligustrifolia A.Juss. & & & & & & & & & & 3 & 6 & \\
\hline \multicolumn{13}{|l|}{ Malvaceae } \\
\hline $\begin{array}{l}\text { Bastardiopsis densiflora (Hook. \& Arn.) } \\
\text { Hassler }\end{array}$ & & & & & & & & & & & 5 & 31 \\
\hline Luehea divaricata Mart. & & 11 & 4 & 8 & 2 & 1 & 1 & 7 & 1 & & & \\
\hline Sida rhombifolia L. & & & 2 & & & & & & & & & \\
\hline
\end{tabular}

\section{Melastomataceae}

Huberia semiserrata DC.

Leandra australis (Cham.) Cogn.

3

Leandra cf. dasytricha (A. Gray) Cogn.

Leandra sp1

Leandra sp2

Miconia cabucu Hoehme

Miconia cf. latecrenata (DC.) Naudin

Miconia cinnamomifolia (DC.) Naudin

Miconia cubatanensis Hoehne

Miconia flammea Cesar.

Miconia ligustroides (DC.) Naudin

Miconia rigidiuscula Cogn.

Miconia sp1

Mouriri chamissoniana Cogn.

Tibouchina cf. trichopoda Baill.

Tibouchina pulchra Cogn.

Tibouchina sellowiana (Cham.) Cogn.

\section{Meliaceae}

Cabralea canjerana (Vell.) Mart.

Cedrela fissilis Vell.

Guarea macrophylla Vahl

Trichilia catigua A.Juss.

Trichilia elegans A.Juss.

Trichilia lepidota Mart.

\begin{tabular}{lllll} 
& 10 & 29 & \\
217 & 3 & 13 & \\
6 & 31 & 16 & 6 \\
41 & 39 & 41 & 1 \\
2 & 76 & 109 & 31 \\
& 4 & 8 & \\
7 & 444 & 197 & 23 \\
2 & & 4 & 5 \\
& 5 & & \\
1 & 7 & & \\
11 & 63 & 97 & 3 \\
& 3 & 7 & 1 \\
& & 3 & 2 \\
120 & 21 & 27 & \\
44 & 211 & 126 & 43 \\
6 & 35 & 10 & \\
\hline
\end{tabular}

\begin{tabular}{|c|c|c|c|c|c|c|c|c|c|c|c|}
\hline & 2 & 6 & 13 & & & 4 & 1 & & & 14 & 24 \\
\hline \multirow[t]{5}{*}{1} & 2 & 19 & 19 & 1 & 5 & 5 & 4 & & 3 & 2 & 1 \\
\hline & & 1 & 1 & & & & & 1 & 4 & 20 & 26 \\
\hline & & 4 & 2 & & & & & & & & \\
\hline & & 7 & 13 & & & 2 & 5 & & 1 & 7 & 5 \\
\hline & & & & & & & & & 4 & 6 & \\
\hline
\end{tabular}

Monimiaceae

Mollinedia sp.

Mollinedia triflora (Spreng.) Tul

$\begin{array}{lllll}1 & & & 3 & 15 \\ & 2 & 3 & 13 & 26\end{array}$

Moraceae

Brosimum lactescens (S. Moore) C.C.

Berg

Ficus cf. insipida Willd.

Ficus organensis (Miq.) Miquel 
TABle 5: Continued.

\begin{tabular}{|c|c|c|c|c|c|c|c|c|c|c|c|c|}
\hline \multirow{2}{*}{ Botanic family/species } & \multicolumn{4}{|c|}{ SDF } & \multicolumn{4}{|c|}{ MOF } & \multicolumn{4}{|c|}{ DOF } \\
\hline & SR & ST & $\mathrm{AR}$ & $\mathrm{AA}$ & SR & ST & $\mathrm{AR}$ & $\mathrm{AA}$ & SR & ST & AR & $\mathrm{AA}$ \\
\hline Maclura tinctoria (L.) D.Don ex Steud. & & & 1 & & & & & & & & 4 & 4 \\
\hline $\begin{array}{l}\text { Sorocea bonplandii (Bail.) Burg., Lanj } \\
\text { \& Boer }\end{array}$ & & & 4 & 22 & & & 9 & & & & & 4 \\
\hline \multicolumn{13}{|l|}{ Myristicaceae } \\
\hline Virola bicuhyba Schott & & & & & & & & & 1 & & 9 & 24 \\
\hline \multicolumn{13}{|l|}{ Myrsinaceae } \\
\hline Myrsine cf. lancifolia Mart. & & & & & & & 1 & & & & & \\
\hline $\begin{array}{l}\text { Myrsine coriacea (Swartz) R. Brown ex } \\
\text { Roemer \& Schultz }\end{array}$ & 1 & 3 & & 3 & 21 & 35 & 52 & 12 & 116 & 277 & 71 & 7 \\
\hline Myrsine parviflora A. DC. & & & & & & & & & 1 & 1 & 6 & 5 \\
\hline Myrsine umbellata Mart ex. DC. & 2 & 2 & 11 & 7 & 6 & & 22 & 7 & 2 & 19 & 12 & 8 \\
\hline \multicolumn{13}{|l|}{ Myrtaceae } \\
\hline $\begin{array}{l}\text { Blepharocalyx salicifolius (Kunth) O. } \\
\text { Berg }\end{array}$ & & & & & & & 1 & 2 & & & & \\
\hline Calyptrantes sp. & & & & & & & & 2 & 1 & & & \\
\hline Calyptranthes cf. grandifolia O. Berg. & & & & & & & & & & & & 9 \\
\hline $\begin{array}{l}\text { Campomanesia guaviroba (DC.) } \\
\text { Kaiaerskou }\end{array}$ & & & & & & & & & & 1 & & 2 \\
\hline $\begin{array}{l}\text { Campomanesia guazumifolia } \\
\text { (Cambess.) O. Berg }\end{array}$ & & & 3 & 2 & & & & & & & & \\
\hline Campomanesia reitziana Legr. & & & & & & & 1 & 1 & & 1 & & 3 \\
\hline Campomanesia sp. & & & & & & & & & & & & 4 \\
\hline Campomanesia xanthocarpa O. Berg & & & & 14 & & & 3 & 7 & & 2 & & 4 \\
\hline Eugenia burkartiana (Legr.) Legr. & & & & & & & & & & 1 & & \\
\hline Eugenia chlorophylla O. Berg & & & & & & & & 2 & & & & \\
\hline Eugenia involucrata DC. & & & & & & & 2 & 2 & & & 1 & 2 \\
\hline Eugenia neoaustralis Sobral & & & & & & & & & & & 1 & \\
\hline Eugenia playtsema O.Berg & & & & & & & & & & & 1 & 2 \\
\hline Eugenia pyriformis Camb. & 1 & & 3 & 5 & & & & & & & & \\
\hline Eugenia uniflora L. & & & & 1 & & & & 2 & 1 & & & \\
\hline Gomidesia palustris (DC.) D.Legrand & & & & & & & & & & & & 11 \\
\hline Gomidesia schaueriana O.Berg & & & & & & & & & & & & 1 \\
\hline Gomidesia spectabilis (DC.) O. Berg & & & & & & & & & 2 & 5 & 13 & 19 \\
\hline Gomidesia tijucensis (Kiaerskov) Legr. & & & & & & & & & & & 1 & 1 \\
\hline $\begin{array}{l}\text { Marlieria cf. eugeniopsioides (Kaus. \& } \\
\text { Legr.) Legr. }\end{array}$ & & & & & & & & & & 3 & 25 & 38 \\
\hline Marlierea parviflora O. Berg. & & & & & & & & & & & & 4 \\
\hline Marlierea tomentosa Cambess. & & & & 1 & & & & & 1 & & 20 & 23 \\
\hline $\begin{array}{l}\text { Mosiera prismatica (D. Legrand) } \\
\text { Landrum }\end{array}$ & & & & & & & 3 & 17 & & & & \\
\hline Myrceugenia sp. & & & & & & & & & & & & 1 \\
\hline Myrcia gracilis O.Berg & & & & & & & & & & 2 & 2 & \\
\hline Myrcia guianensis (Aubl.) DC. & & & & & 1 & & & 2 & & & & \\
\hline Myrcia multiflora (Lam.) DC. & & & & & & & 1 & & & & 2 & \\
\hline Myrcia palustris DC. & & & & & & & & 1 & & & & \\
\hline Myrcia rostrata DC. & & & & & & & 17 & 3 & 3 & 3 & 22 & 10 \\
\hline Myrcia splendens (Sw.) DC. & & & & & & & & & & & 1 & \\
\hline Myrcia tenuivenosa Kiaersk. & & & & & & & & & & & 3 & \\
\hline
\end{tabular}


Table 5: Continued.

\begin{tabular}{|c|c|c|c|c|c|c|c|c|c|c|c|c|}
\hline \multirow{2}{*}{ Botanic family/species } & \multicolumn{4}{|c|}{ SDF } & \multicolumn{4}{|c|}{ MOF } & \multicolumn{4}{|c|}{ DOF } \\
\hline & SR & ST & $\mathrm{AR}$ & $\mathrm{AA}$ & SR & ST & AR & $\mathrm{AA}$ & SR & ST & AR & $\mathrm{AA}$ \\
\hline Myrcianthes pungens (O. Berg) Legr. & & & & & & & & 1 & & & & \\
\hline Myrciaria tenella (DC.) O. Berg. & & & & 1 & & & 2 & 2 & & & & 2 \\
\hline Myrtaceae sp1 & & & & & & & 2 & 20 & & & & \\
\hline Myrtaceae sp2 & & & & & & & & 21 & & & & 1 \\
\hline Myrtaceae sp3 & & 2 & & & & & & & & & & \\
\hline Myrtaceae sp4 & & & & & & & & & & & 2 & 10 \\
\hline Psidium cattleyanum Sabine & & & & & & & & 1 & 9 & 32 & 10 & 4 \\
\hline \multicolumn{13}{|l|}{ Nyctaginaceae } \\
\hline Guapira hirsuta (Choisy) Lundell & & & & & & & & & & & 14 & \\
\hline Guapira opposita (Vell.) Reitz & & & & & & & & & & 3 & 24 & 30 \\
\hline Pisonia ambigua Heimerl & & & 1 & 4 & & & & & & 1 & 4 & 5 \\
\hline \multicolumn{13}{|l|}{ Ochnaceae } \\
\hline Ouratea parviflora (DC.) Baill. & & & & & & & & & & 1 & 9 & 16 \\
\hline \multicolumn{13}{|l|}{ Oleaceae } \\
\hline $\begin{array}{l}\text { Chionanthus filiformis (Vell.) P.S. } \\
\text { Green }\end{array}$ & & & & & & & & & 1 & & & \\
\hline \multicolumn{13}{|l|}{ Olacaceae } \\
\hline Heisteria silvianii Schwacke & & & & & & & & & & 1 & 1 & 2 \\
\hline \multicolumn{13}{|l|}{ Phytolaccaceae } \\
\hline Phytolacca dioica $\mathrm{L}$. & & 6 & & 2 & & & & & & & & \\
\hline Seguieria glaziovii Briq. & & 1 & & & & & & & & & & \\
\hline \multicolumn{13}{|l|}{ Picramniaceae (Simaroubaceae) } \\
\hline Picramnia parvifolia Engl. & & & & & & & 2 & & & & & \\
\hline Picramnia sp. & & & & 3 & & & & & & & & \\
\hline \multicolumn{13}{|l|}{ Piperaceae } \\
\hline Piper arboreum Aubl & & & & & & & & & & 5 & & 5 \\
\hline Piper cernuum Vell. & & & & & & & & & & 5 & 3 & 3 \\
\hline Piper gaudichaudianum Kuntze & & & & & & & & & 4 & 24 & 112 & 28 \\
\hline \multicolumn{13}{|l|}{ Podocarpaceae } \\
\hline Podocarpus lambertii Klotzsch & & & & & & & 18 & & & & & \\
\hline \multicolumn{13}{|l|}{ Polygonaceae } \\
\hline Coccoloba warmingii Meisn. & & & & & & & & & & 1 & 3 & 1 \\
\hline Ruprechtia laxiflora Meisn. & 15 & 1 & & & & & 2 & 2 & & & & \\
\hline \multicolumn{13}{|l|}{ Proteaceae } \\
\hline Roupala asplenioides Sleumer & & & & & & & & 9 & & & & \\
\hline Roupala cataractarum Sleumer & & & 7 & & & & & & & & & \\
\hline Roupala cf. brasiliensis Klotzsch & & & & & & & & & & & & 1 \\
\hline \multicolumn{13}{|l|}{ Quiinaceae } \\
\hline Quiina glaziovii Engl. & & & & & & & & & & 1 & 2 & 11 \\
\hline \multicolumn{13}{|l|}{ Rhamnaceae } \\
\hline Hovenia dulcis Thumb. (exótica) & & & 30 & 16 & & & 1 & & & & & \\
\hline Rhamnaceae sp1 & & 3 & 1 & & & & & & & & & \\
\hline Scutia buxifolia Reissek. & & 1 & & & & & 5 & 7 & & & & \\
\hline
\end{tabular}


TABle 5: Continued.

\begin{tabular}{|c|c|c|c|c|c|c|c|c|c|c|c|c|}
\hline \multirow{2}{*}{ Botanic family/species } & \multicolumn{4}{|c|}{ SDF } & \multicolumn{4}{|c|}{ MOF } & \multicolumn{4}{|c|}{ DOF } \\
\hline & SR & ST & $\mathrm{AR}$ & $\mathrm{AA}$ & SR & ST & $\mathrm{AR}$ & $\mathrm{AA}$ & SR & ST & $\mathrm{AR}$ & $\mathrm{AA}$ \\
\hline \multicolumn{13}{|l|}{ Rosaceae } \\
\hline $\begin{array}{l}\text { Prunus brasiliensis (Cham. \& } \\
\text { Schlechtd) D. Dietr. }\end{array}$ & & & & & & & & & 1 & & 4 & 1 \\
\hline Prunus myrtifolia (1.) Urb. & & & & & & & 5 & & & & & \\
\hline Prunus sellowii Koehne & & & 12 & 1 & & & & & & & 1 & 3 \\
\hline Prunus sp. & & & 1 & & & & & & & & 1 & \\
\hline \multicolumn{13}{|l|}{ Rubiaceae } \\
\hline Alibertia concolor (Cham.) K. Schum. & & & & & & & & & & 1 & 2 & 2 \\
\hline Amaioua guianensis Aubl. & & & & & & & & & & 9 & 9 & 4 \\
\hline $\begin{array}{l}\text { Bathysa australis (A.St.-Hil.) Benth. \& } \\
\text { Hook.F. }\end{array}$ & & & & & & & & & 4 & 3 & 24 & 15 \\
\hline Chomelia pedunculosa Bentham & & & & & & & & & & & 1 & 5 \\
\hline $\begin{array}{l}\text { Posoqueria latifolia (Rudge) Roem. \& } \\
\text { Schult. }\end{array}$ & & & & & & & & & 1 & 2 & 5 & 6 \\
\hline Psychotria carthagenensis Jacq. & & & & & & & & & & & 2 & 4 \\
\hline Psychotria leiocarpa Cham. & & & & & & & & & & 1 & 9 & 7 \\
\hline Psychotria longipes Muell.Arg. & & 1 & & 1 & & & & & & 8 & 62 & 43 \\
\hline $\begin{array}{l}\text { Psychotria nuda (Cham. and Schltdl.) } \\
\text { Wawra }\end{array}$ & & & 2 & & & & & & & & 8 & 10 \\
\hline $\begin{array}{l}\text { Psychotria officinalis (Aubl.) Raeusch. } \\
\text { ex Sandwith }\end{array}$ & & & & 1 & & & & & & & 5 & 14 \\
\hline Psychotria sp1 & & & & & & & 3 & 12 & & & & \\
\hline Psychotria sp2 & & 1 & 3 & 6 & & & & & & & & \\
\hline Psychotria sp3 & & & & & & & & & & & 2 & 3 \\
\hline Psychotria sp4 & & & & & & & & & & 1 & 34 & 45 \\
\hline Psychotria stenocalyx Müll.Arg. & & & & & & & & & & 2 & 9 & 21 \\
\hline Psychotria suterella Müll. Arg. & & & & & & & 2 & 2 & 1 & & 15 & 22 \\
\hline Randia armata (Sw.) DC. & & 5 & 1 & & & & 2 & 2 & & & & \\
\hline Rubiaceae sp1 & & & 3 & 12 & & & & & & & & \\
\hline Rubiaceae sp2 & & & & & & & & & & & & 5 \\
\hline $\begin{array}{l}\text { Rudgea jasminioides (Cham.) } \\
\text { Müll.Arg. }\end{array}$ & & & & & & & & & 1 & 3 & 21 & 25 \\
\hline \multicolumn{13}{|l|}{ Ruscaceae (Agavaceae) } \\
\hline Cordyline dracaenoides Kunth & & & & & 3 & & 1 & 7 & & & & \\
\hline
\end{tabular}

\section{Rutaceae}

Balfourodendron riedelianum (Engl.)

Engl.

45

Esenbeckia grandiflora Mart.

Helietta apiculata Benth.

Pilocarpus pennatifolius Lem.

Zanthoxylum cf. astrigera Cowan

Zanthoxylum hiemalis A.St.-Hil.

Zanthoxylum petiolare A.St.-Hil. \& Tul.

5

Sabiaceae

Meliosma cf. sellowii Urban.

Salicaceae (Flacourtiaceae)

Banara parviflora (A. Gray) Benth. 2 
TABle 5: Continued.

\begin{tabular}{|c|c|c|c|c|c|c|c|c|c|c|c|c|}
\hline \multirow{2}{*}{ Botanic family/species } & \multicolumn{4}{|c|}{ SDF } & \multicolumn{4}{|c|}{ MOF } & \multicolumn{4}{|c|}{ DOF } \\
\hline & SR & ST & $\mathrm{AR}$ & $\mathrm{AA}$ & SR & ST & AR & $\mathrm{AA}$ & SR & ST & $\mathrm{AR}$ & $\mathrm{AA}$ \\
\hline Casearia decandra Jacq. & & 6 & 5 & 4 & 2 & 7 & 21 & 22 & 2 & & 33 & 31 \\
\hline Casearia sylvestris Sw. & 2 & 1 & 19 & 29 & & & 4 & 8 & 8 & 5 & 67 & 23 \\
\hline Xylosma pseudosalzmannii Sleumer. & & & & & & & 1 & 1 & & & & \\
\hline \multicolumn{13}{|l|}{ Sapindaceae } \\
\hline $\begin{array}{l}\text { Allophylus edulis (A.St.-Hil. \& et al.) } \\
\text { Radlk }\end{array}$ & 1 & 4 & 9 & 39 & & & 19 & 23 & & & 20 & 15 \\
\hline $\begin{array}{l}\text { Allophylus guaraniticus (A.St.-Hil.) } \\
\text { Radlk }\end{array}$ & & & & & & 3 & 36 & 26 & & & & \\
\hline Cupania oblongifolia Mart. & & & & & & & & & & 2 & 2 & \\
\hline Cupania vernalis Camb. & 2 & 13 & 34 & 65 & 4 & 9 & 24 & 75 & 22 & 10 & 51 & 7 \\
\hline Diatenopteryx sorbifolia Radlk. & & & 5 & & & & & & & & & \\
\hline Dodonaea viscosa (L.) Jacq. & & & & & & & & & 127 & 18 & 1 & \\
\hline Matayba elaeagnoides Radlk. & & 6 & 15 & 25 & 28 & 30 & 93 & 140 & & & & \\
\hline Matayba guianensis Aubl. & & & & & & & & & 4 & 3 & 21 & 8 \\
\hline \multicolumn{13}{|l|}{ Sapotaceae } \\
\hline $\begin{array}{l}\text { Chrysophyllum gonocarpum (Mart. \& } \\
\text { Eichl.)Engl. }\end{array}$ & 1 & & 6 & 8 & & & & & & & & \\
\hline $\begin{array}{l}\text { Chrysophyllum marginatum (Hook. \& } \\
\text { Eichler) Engl. }\end{array}$ & 1 & 3 & 10 & 11 & & & & & & & & 1 \\
\hline Pouteria venosa (Mart.) Baehni & & & & & & & & & & 2 & 8 & 3 \\
\hline \multicolumn{13}{|l|}{ Solanaceae } \\
\hline Capsicum cf. flexuosum Sendtn & & & & & & & 2 & & & & & \\
\hline Cestrum strigilatum Ruiz \& Pav. & 1 & & 4 & & & & & & & & & \\
\hline Solanaceae sp1 & & & & & & & 4 & & & & & \\
\hline Solanum diflorum Vell. & 9 & 29 & 2 & 1 & 11 & 34 & 1 & & 6 & 9 & 2 & \\
\hline Solanum mauritianum Scopoli & & 1 & 3 & 6 & 4 & 2 & & & & & & \\
\hline Solanum pseudoquina A.St.-Hil. & & & 2 & 7 & & & 1 & & 1 & & & \\
\hline Solanum sanctae-catharinae Dunal & & & & & 30 & & 1 & & & & & \\
\hline Solanum sp2 & & & & & & & & & 2 & & & \\
\hline Solanum sp3 & 1 & & 33 & 6 & 1 & & & & 1 & 1 & 24 & 8 \\
\hline \multicolumn{13}{|l|}{ Styracaceae } \\
\hline Styrax leprosus Hook. \& Arn. & & 1 & & 1 & & & 2 & 2 & & & & \\
\hline \multicolumn{13}{|l|}{ Symplocaceae } \\
\hline Symplocos celastrina Mart. & & & & & & & 2 & & & & & \\
\hline Symplocos lanceolata (Mart.) A.DC. & & & & & & & & & & & 1 & \\
\hline Symplocos sp1 & & & & & & & 5 & & & & 4 & 3 \\
\hline Symplocos tenuifolia Brand & & & 3 & & & & 2 & 5 & & & & \\
\hline Symplocos uniflora (Pohl) Benth. & & & & & & & & & & 1 & 2 & \\
\hline \multicolumn{13}{|l|}{ Theaceae } \\
\hline Laplacea fruticosa (Schrad.) Kobuski. & & & & & & & & & & 4 & 8 & \\
\hline \multicolumn{13}{|l|}{ Urticaceae } \\
\hline Boehmeria caudata Sw & 13 & 30 & 7 & & & & & & & & & \\
\hline Cecropia glaziovii Snethl. & & & & & & & & & 33 & 28 & 10 & 2 \\
\hline Urera baccifera (L.) Gaudich. ex Wedd & & 3 & & & & & & & & & & \\
\hline \multicolumn{13}{|l|}{ Verbenaceae } \\
\hline Aloysia virgata (Ruiz \& Pav.) Juss. & 1 & 2 & 31 & 1 & & & & & & & & \\
\hline Citharexylum myrianthum Cham. & & & & & & & & & & 3 & & 1 \\
\hline Lantana camara L. & & & & & & & & & & & 2 & 1 \\
\hline
\end{tabular}


TABle 5: Continued.

\begin{tabular}{|c|c|c|c|c|c|c|c|c|c|c|c|c|}
\hline \multirow{2}{*}{ Botanic family/species } & \multicolumn{4}{|c|}{ SDF } & \multicolumn{4}{|c|}{ MOF } & \multicolumn{4}{|c|}{ DOF } \\
\hline & SR & ST & $\mathrm{AR}$ & AA & SR & ST & $\mathrm{AR}$ & $\mathrm{AA}$ & SR & ST & $\mathrm{AR}$ & $\mathrm{AA}$ \\
\hline \multicolumn{13}{|l|}{ Winteraceae } \\
\hline Drimys brasiliensis Miers & & & & & & & 8 & 9 & & & & \\
\hline \multicolumn{13}{|l|}{ Undetermined } \\
\hline Nonidentified & & 3 & 6 & 1 & & & 10 & 23 & & 7 & 6 & 15 \\
\hline \multicolumn{13}{|l|}{ Dead } \\
\hline Dead & 6 & 5 & 12 & 15 & & & 9 & 7 & & 13 & 6 & 1 \\
\hline
\end{tabular}

\section{Acknowledgments}

The authors gratefully acknowledge the expert help of Dr. Marcos Sobral and Prof. Dr. Ademir Reis in the taxonomic identification of forest species. They thank the Núcleo de Pesquisas em Florestas Tropicais for the support. Financial support came from the Conselho Nacional de Desenvolvimento Científico e Tecnológico (CNPq, Brazil) and from the Fundação de Apoio À Pesquisa Científica e Tecnológica do Estado de Santa Catarina (FAPESC).

\section{References}

[1] J. Benitez-Malvido, "Impact of forest fragmentation on seedling abundance in a tropical rain forest," Conservation Biology, vol. 12, no. 2, pp. 380-389, 1998.

[2] R. A. Mittermeier, N. Myers, J. B. Tliomsen, and S. Olivieri, "Biodiversity hotspots and major tropical wilderness areas: approaches to setting conservation priorities," Conservation Biology, vol. 12, no. 3, pp. 516-520, 1998.

[3] I. M. Turner and R. T. Corlett, "The conservation value of small, isolated fragments of lowland tropical rain forest," Trends in Ecology and Evolution, vol. 11, no. 8, pp. 330-333, 1999.

[4] P. A. Zuidema, J. A. Sayer, and W. Dijkman, "Forest fragmentation and biodiversity: the case for intermediate-sized conservation areas," Environmental Conservation, vol. 23, no. 4, pp. 290-297, 1996.

[5] K. H. Redford and B. D. Richter, "Conservation of biodiversity in a world of use," Conservation Biology, vol. 13, no. 6, pp. 1246-1256, 1999.

[6] R. A. Kramer and C. P. van Schaik, "Preservation paradigms and tropical rain forests," in Last Stand: Protected Areas and the Defense of Tropical Biodiversity, R. A. Kramer, C. P. van Schaik, and J. Johnson, Eds., pp. 3-14, Oxford University Press, New York, NY, USA, 1997.

[7] R. E. Bodmer, J. W. Penn, P. Puertas, I. L. Moya, and T. G. Fang, "Linking conservation and local people through sustainable use of natural resources," in Harvesting Wild Species, C. H. Freese, Ed., pp. 315-358, Johns Hopkins, Baltimore, Md, USA, 1997.

[8] Fundação SOS Mata Atlântica e do Instituto Nacional de Pesquisas Espaciais, "Atlas dos remanescentes Florestais da Mata Atlântica Período 2000-2005,” São Paulo, Brazil, 2008.

[9] M. H. Queiroz, Approche phytoécologoque et dynamique des formations végetales secondairesdéveloppées après abandon dês activités agricoles, dans lê domaine de la fôret ombrophile dense de versant (Fôret Atlantique) à Santa Catarina-Brésil, Ph.D. thesis, Tese (Doutorado)—École Nationale du Génie Rural, des Eaux et des Forêts, Nancy, França, 1994.
[10] A. Gómez-Pompa, "Possible papel de la vegetación secundária en la evolución de la flora tropical," Biotropica, vol. 3, pp. 125135, 1971.

[11] C. Uhl, "Factors controlling succession following slash-andburn agriculture in Amazonia," Journal of Ecology, vol. 75, no. 2, pp. 377-407, 1987.

[12] C. Uhl, R. Buschbacher, and E. A. S. Serrao, "Abandoned pastures in eastern Amazonia. I. Patterns of plant succession," Journal of Ecology, vol. 76, no. 3, pp. 663-681, 1988.

[13] J. G. Saldarriaga, D. C. West, M. L. Tharp, and C. Uhl, "Longterm chronosequence of forest succession in the upper Rio Negro of Colombia and Venezuela," Journal of Ecology, vol. 76, no. 4, pp. 938-958, 1988.

[14] B. Finegan, "Pattern and process in neotropical secondary rain forests: the first 100 years of succession," Trends in Ecology and Evolution, vol. 11, no. 3, pp. 119-124, 1996.

[15] T. C. Whitmore, An Introduction to Tropical Rain Forests, Oxford University Press, New York, NY, USA, 2nd edition, 1998.

[16] M. R. Guariguata and R. Ostertag, "Neotropical secondary forest succession: changes in structural and functional characteristics," Forest Ecology and Management, vol. 148, no. 1-3, pp. 185-206, 2001.

[17] A. Siminski and A. C. Fantini, "Roça-de-toco: uso de recursos florestais e dinâmica da paisagem rural no litoral de Santa Catarina," Ciencia Rural, vol. 37, no. 3, pp. 1-10, 2007.

[18] R. M. Klein, "Ecologia da flora e vegetação do Vale do Itajaí," Sellowia, vol. 32, pp. 164-369, 1980.

[19] P. Y. Kageyama, A. Reis, and A. A. Carpanezzi, "Potencialidades e restrições da regeneração artificial na recuperação de áreas degradadas," in Simposio Nacional de Recuperação de Áreas Degradadas, pp. 1-7, Livro de resumos do Simpósio Nacional de Recuperação de Áreas Degradadas, 1992.

[20] H. P. Velso, A. L. R. R. Rangel Filho, and J. C. A. LIMA, "Classificação da vegetação brasileira, adaptada a um sistema universal," Rio de Janeiro, Brazil, IBGE, Departamento de Recursos Naturais e Estudos Ambientais, 1991.

[21] B. Rambo, "Der Regenwald am oberen Uruguay," Sellowia, vol. 7, pp. 183-223, 1956.

[22] B. Rambo, A Fisionaomiado Rio Grande do Sul: Ensaio de Monografia Natural, UNISINOS, São Leopoldo, Brazil, 3rd edition, 1994.

[23] R. M. Klein, “Árvores nativas da floresta Subtropical do Alto Uruguai,” Sellowia, vol. 24, pp. 9-62, 1972.

[24] C. A. M. Lindman, A Vegetação no Rio Grande do Sul, Itatiaia, São Paulo, Brazil, 2nd edition, 1974.

[25] R. M. Klein, Mapa Fitogeográfico do Estado de Santa Catarina, Herbário Barbosa Rodrigues, Itajaí, Brazil, 1978. 
[26] A. Reis, Manejo e conservação das florestas catarinenses, M.S. thesis, UFSC, Florianópolis, Brazil, 1993, Dissertação (Concurso para Professor Titular)_Universidade Federal de Santa Catarina, 1993.

[27] B. Bremer, K. Bremer, M. W. Chase et al., "An update of the Angiosperm Phylogeny Group classification for the orders and families of flowering plants: APG II," Botanical Journal of the Linnean Society, vol. 141, no. 4, pp. 399-436, 2003.

[28] G. J. Shepherd, "FITOPAC 1. Manual do usuario," Campinas, Brazil. Departamento de Botânica. UNICAMP, 1994.

[29] R. K. Colwell, “EstimateS 7. 5 user's guide," Storrs, EUA: Department of Ecology \& Evolutionary Biology, University of Connecticut, 2006, http://www.viceroy.eeb.uconn.edu/ estimateS.

[30] StatSoft, INC., "STATISTICA (data analysis software system), version 6," 2001, http://www.statsoft.com.

[31] T. M. Aide, J. K. Zimmerman, L. Herrera, M. Rosario, and M. Serrano, "Forest recovery in abandoned tropical pastures in Puerto Rico," Forest Ecology and Management, vol. 77, no. 1-3, pp. 77-86, 1995.

[32] T. Mitche, J. K. Zimmerman, J. B. Pascarella, L. Rivera, and H. Marcano-Vega, "Forest regeneration in a chronosequence of tropical abandoned pastures: implications for restoration ecology," Restoration Ecology, vol. 8, no. 4, pp. 328-338, 2000.

[33] M. Tabarelli and W. A. Mantovani, "A regeneração de uma floresta tropical montana após corte e queima (São PauloBrasil)," Revista Brasileira de Biologia, vol. 59, no. 2, pp. 239 250, 1999.

[34] R. R. Oliveira, "Ação antrópica e resultantes sobre a estrutura e composição da Mata Atlântica na Ilha Grande, RJ," Rodriguésia, vol. 53, no. 82, pp. 33-58, 2002.

[35] D. Sheil and D. F. R. P. Burslem, "Disturbing hypotheses in tropical forests," Trends in Ecology and Evolution, vol. 18, no. 1, pp. 18-26, 2003.

[36] P. Legendre and L. Legendre, Numerical Ecology, Elsevier, Amsterdam, The Netherlands, 2nd edition, 1998.

[37] A. E. Magurran, Measuring Biological Diversity, Blackwell, Oxford, UK, 2004.

[38] J. M. D. Torezan, Estudo da sucessão secundária na Floresta Ombrófila Densa Submontana, em áreas anteriormente cultivadas pelo sistema de "coivara", em Iporanga, SP, Dissertação de Mestrado, Departamento de Botânica/ UFPR, Curitiba, Brazil, 1995.

[39] S. V. A. Pessoa, R. R. Guedes-Bruni, and C. K. Bruno, "Composição florística e estrutura do componente arbustivoarbóreo de um trecho secundário de floresta montana na Reserva Ecológica de Macaé de Cima," in Serra de Macaé de Cima: Diversidade Florística e Conservação em Mata Atlântica, H. C. de Lima and R. R. Guedes-Bruni, Eds., pp. 147-168, Jardim Botânico do Rio de Janeiro, Rio de Janeiro, Brazil, 1997.

[40] A. F. Silva and H. F. Leitão-Filho, "Composição florística e estrutura de um trecho de mata atlântica de encosta no município de Ubatuba (São Paulo, Brasil)," Revista Brasileira de Botânica, vol. 5, no. 132, pp. 43-52, 1982.

[41] H. C. Rodrigues, Composição florística e fitossociologia de um trecho de mata atlântica na Reserva Biológica do Tinguá, Rio de Janeiro, RJ, Dissertação de Mestrado, MN/UFRJ, Rio de Janeiro, Brazil, 1996.

[42] S. J. Longhi, Agrupamento e análise fitossociológica de comunidades florestais na subbacia hidrográfica do rio Passo Fundo$R S$, Ph.D. thesis, Tese (Doutorado em Ciências Florestais) Universidade Federal do Paraná, Setor de Ciências Agrárias, Curitiba, Brazil, 1997.
[43] M. C. Dias, A. O. S. Vieira, J. N. Nakajima, J. A. Pimenta, and P. Carneiro Lobo, "Composição florística e fitossociológica do componente arbóreo das florestas ciliares do rio Iapó, na bacia do rio Tibagi, Tibagi, PR," Revista Brasileira de Botânica, vol. 21, pp. 183-195, 1998.

[44] M. M. R. F. Melo, R. J. Oliveira, 1. Rossi, M. C. H. Mamede, and I. Cordeiro, "Fitossociologia de trecho de mata atlântica na planície do Rio Verde, Estação Ecológica de Juréia-Itatins, SP, Brasil," in IV Simpósio de Ecossistemas da Costa Sul e Sudeste Brasileira Estrutura, Função e Manejo, vol. 1, pp. 49-56, Águas de Lindóia, Anais, Brazil, 1998.

[45] N. M. Ivanauskas, R. R. Rodrigues, and A. G. Nave, "Phytosociology of the Semi-deciduous Seasonal Forest fragment in Itatinga, São Paulo, BrazilFitossociologia de um trecho de Floresta Estacional Semidecidual em Itatinga, São Paulo, Brasil," Scientia Forestalis, vol. 56, pp. 83-99, 1999.

[46] A. R. Ruschel, R. O. Nodari, and B. M. Moerschbacher, "Woody plant species richness in the Turvo State park, a large remnant of deciduous Atlantic forest, Brazil," Biodiversity and Conservation, vol. 16, no. 6, pp. 1699-1714, 2007.

[47] R. H. Whittaker, "Evolution and measurement of species diversity," Taxon, vol. 21, pp. 213-251, 1972.

[48] A. E. Magurran, Ecological Diversity and Its Measurement, Croom Helm, London, UK, 1988.

[49] F. R. Martins and F. A. M. Santos, "Técnicas usuais de estimativa da biodiversidade," Revista Holos, vol. 1, no. 1, pp. 236-267, 1999.

[50] S. Vaccaro and S. J. Longhi, "Análise fitossociológica de algumas áreas remanescentes da Floresta do Alto Uruguai entre os rios Ijuí e Turvo, no Rio Grande do Sul," Ciência Florestal, vol. 5, pp. 33-53, 1995.

[51] R. M. Rondon-Neto, S. A. Botelho, M. A. L. Fontes, A. C. Davide, and J. M. R. Faria, "Estrutura e composição florística da comunidade arbustivo arbórea de uma clareira de formação antrópica, em uma floresta estacional semidecídua montana," Cerne, vol. 6, no. 2, pp. 79-94, 2000.

[52] C. Andreis, S. J. Longhi, E. J. Brun et al., "Estudo fenológico em três fases sucessionais de uma Floresta Estacional Decidual no município de Santa Tereza, RS," Revista Árvore, vol. 29, no. 1, pp. 55-53, 2005.

[53] C. Hack, S. J. Longhi, A. A. Boligon, A. B. Murari, and D. T. Pauleski, "Analise fitossociológica de um fragmento de Floresta Estacional Decidual no município de Jaguari, RS," Ciência Rural, vol. 35, no. 5, pp. 1083-1091, 2005.

[54] R. M. Rondon Neto, C. Kozera, R. R. Andrade et al., "Caracterização florística e estrutural de um fragmento de Floresta Ombrófila Mista, em Curitiba, PR—Brasil," Floresta, vol. 32, no. 1, pp. 3-16, 2002.

[55] T. Pezzat, A. Wagner, C. Wisniewski, C. R. Sanquetta, and P. T. L. Pires, "Análise estrutural comparada entre floras arbóreas de fragmentos florestais ao longo da margem do reservatório de Salto Caxias PR," in VI Congresso de Ecologia do Brasil, vol. 1, pp. 501-503, Anais, Ceará, Brazil, 2003.

[56] I. S. Narvaes, D. A. Brena, and S. J. Longhi, "Estrutura da regeneração natural em Floresta Ombrófila Mista na Floresta Nacional de São Francisco de Paula, RS," Ciências Florestais, vol. 15, no. 4, pp. 331-342, 2005.

[57] A. J. K. Ramos and E. Boldo, "Diversidade florística e aspectos fitossociológicos de formações florestais em estágio sucessional secundário na floresta ombrófila mista, município de Caxias do Sul-RS," Revista Brasileira de Agroecologia, vol. 2, no. 1, pp. 111-116, 2007. 
[58] A. T. Oliveira-Filho, D. A. Carvalho, M. A. L. Fontes, E. van den Berg, N. Curi, and W. A. C. Carvalho, "Variações estruturais do compartimento arbóreo de uma floresta semidecídua alto-montana na chapada das Perdizes, Carrancas, MG," Revista Brasileira de Botânica, vol. 27, pp. 291-309, 2004.

[59] M. Mantovani, A. R. Ruschel, Â. Puchalski, J. Z. Da Silva, M. S. Dos Reis, and R. O. Nodari, "Diversidade de espécies e estrutura sucessional de uma formação secundária da floresta ombrófila densa," Scientia Forestalis/Forest Sciences, no. 67, pp. 14-26, 2005.

[60] L. A. Schorn and F. Galvão, "Dinâmica da regeneração natural em três estágios sucessionais de uma floresta ombrófila densa em Blumenau, SC," Floresta, vol. 36, no. 1, pp. 59-74, 2006.

[61] D. Liebsch, R. Goldenberg, and M. C. M. Marques, "Florística e estrutura de comunidades vegetais em uma cronoseqüência de Floresta Atlântica no Estado do Paraná, Brasil," Acta Botanica Brasilica, vol. 21, no. 4, pp. 983-992, 2007. 

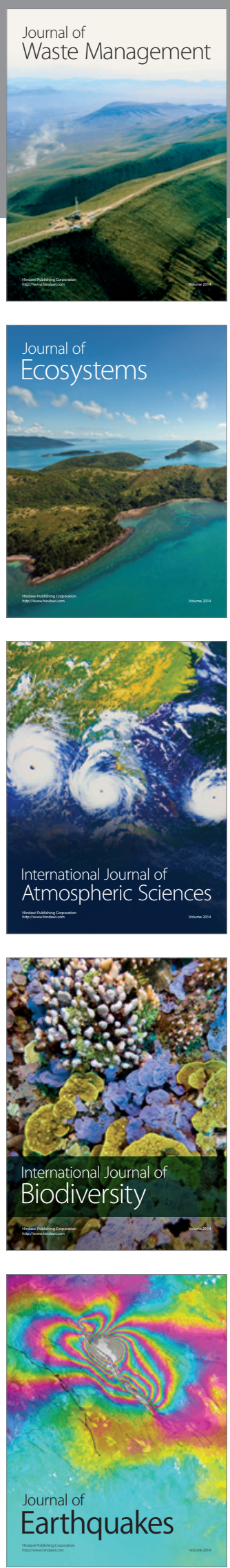
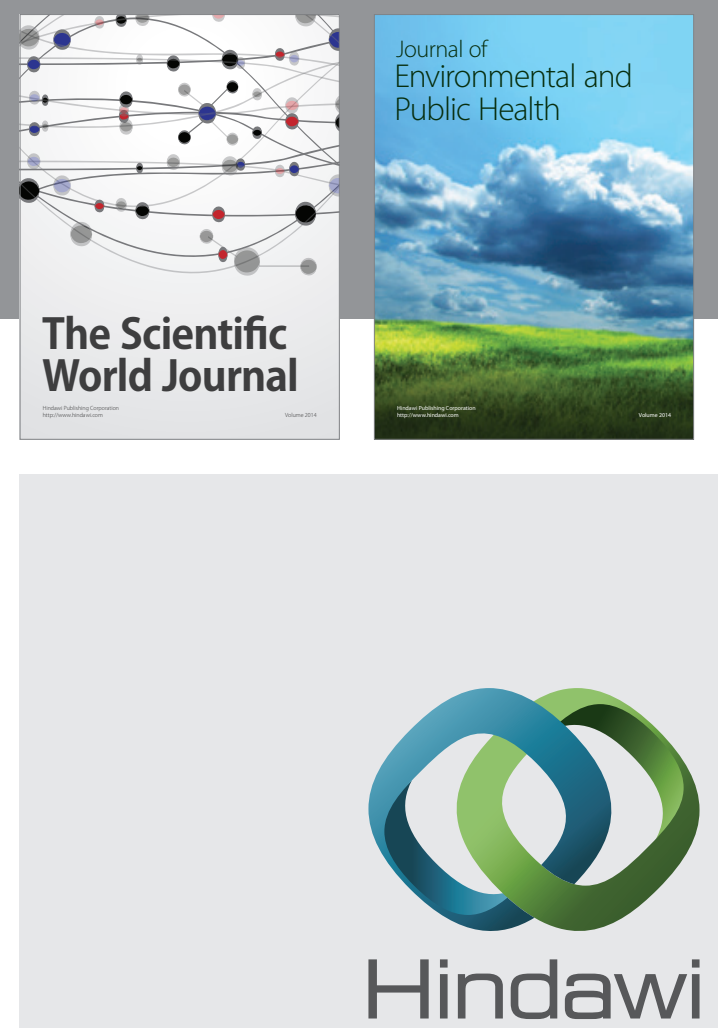

Submit your manuscripts at

http://www.hindawi.com
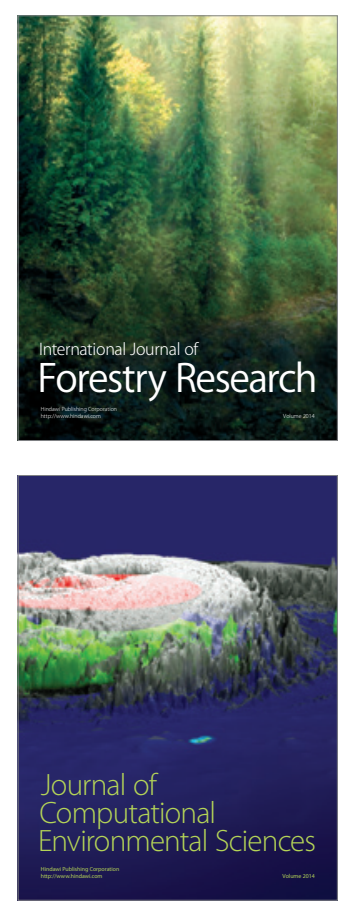
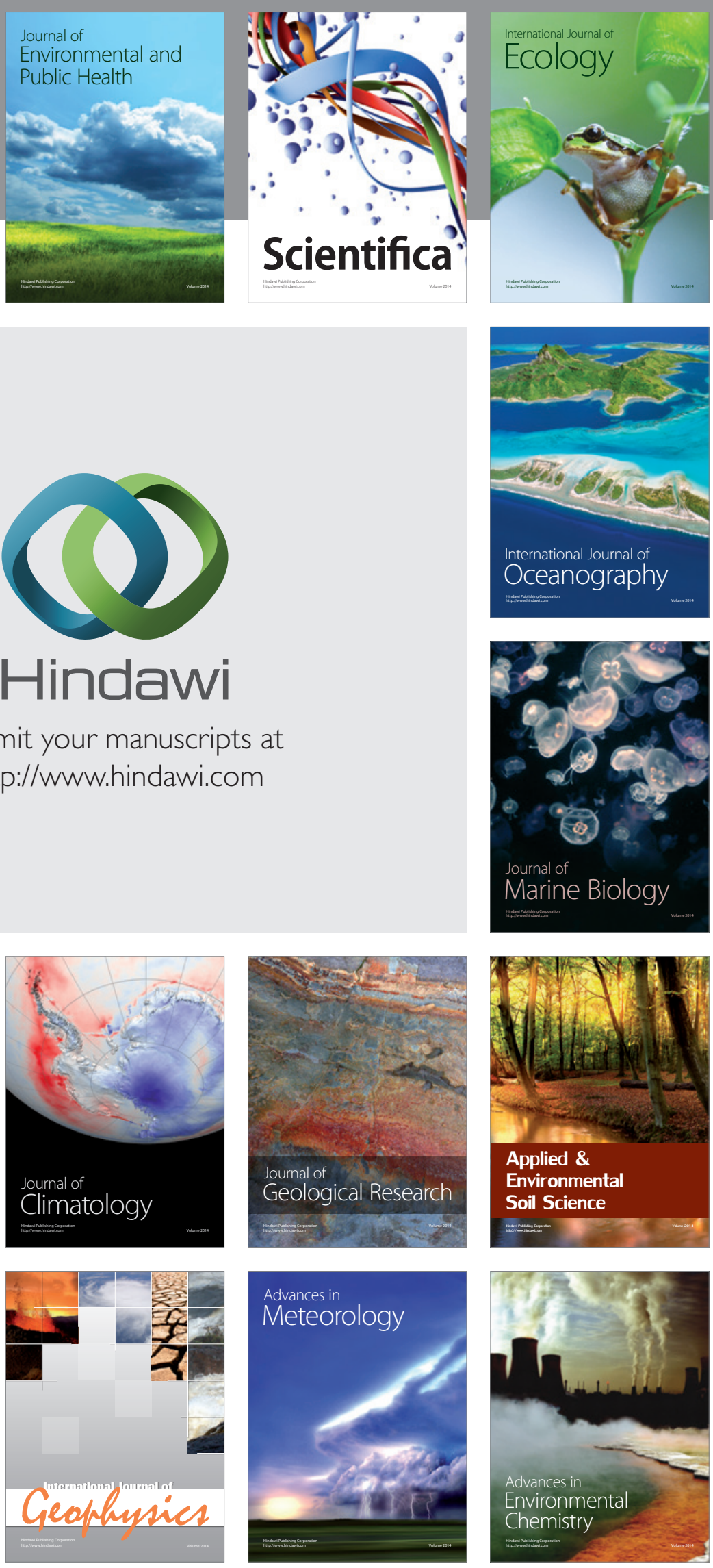